\title{
MATHEMATICAL AND NUMERICAL ANALYSIS OF AN ALTERNATIVE WELL-POSED TWO-LAYER TURBULENCE MODEL*
}

\author{
Bijan Mohammadi ${ }^{1}$ and Guillaume Puigt ${ }^{1}$
}

\begin{abstract}
In this article, we wish to investigate the behavior of a two-layer $k-\varepsilon$ turbulence model from the mathematical point of view, as this model is useful for the near-wall treatment in numerical simulations. First, we explain the difficulties inherent in the model. Then, we present a new variable $\theta$ that enables the mathematical study. Due to a problem of definition of the turbulent viscosity on the wall boundary, we consider an alternative version of the original equation. We show that some physical aspects of the model are preserved by the new formulation, and in particular, we show how the physicists can help us to prove the existence of a solution of our problem. Finally, we are interested in the Navier-Stokes equations coupled with the modified turbulence model and we show that the alternative model may be preferred to the original one, because of its good properties (existence of a solution of the coupled problems).
\end{abstract}

Résumé. L'objectif de cet article est l'étude du modèle de turbulence $k-\varepsilon$ bi-couche qui est utilisé pour le traitement des régions situées près de la paroi dans les simulations numériques. Dans un premier temps, nous expliquons les difficultés engendrées par le modèle de turbulence bi-couche du point de vue numérique. Nous présentons une nouvelle variable $\theta$ et nous nous intéressons à son équation. L'étude mathématique est présentée et, à cause de la singularité de la viscosité turbulente à la paroi, nous nous intéressons à une version alternative du problème original. Nous vérifions les caractéristiques physiques du modèle de turbulence. En nous inspirant des résultats physiques, l'on déduit des estimations nécessaires pour prouver l'existence de la solution de notre problème initial. Enfin, nous nous intéressons au couplage de ce modèle de turbulence avec les équations de NavierStokes et nous montrons que le modèle alternatif doit être préféré au modèle original (existence d'une solution pour les problèmes couplés).

Mathematics Subject Classification. 35A15, 35F25, 90C30, 76F40, 76F60.

Received: April 2, 2001. Revised: October 2, 2001.

\section{INTRODUCTION}

Nowadays, the two-equations $k-\varepsilon$ turbulence model is one of the most useful turbulence models. Its drawback is to be valid in regions where the local Reynolds number $y^{+}$, defined in (2), is large, which has led physicists to find extensions of this model to account for the low-Reynolds regions existing near wall boundaries [3,17]. In particular, the two-layer $k-\varepsilon$ turbulence model is a way to account for these regions: far from the wall,

Keywords and phrases. Incompressible turbulent flows, two-layer $k-\varepsilon$ model, near-wall treatment.

* Research supported by the French National Agency for Nuclear Energy (CEA).

1 University of Montpellier II, Mathematics Department, ACSIOM Laboratory, France. e-mail: mohamadi@math.univ-montp2.fr and puigt@math.univ-montp2.fr 
we solve two parabolic equations for the kinetic turbulent energy $k$ and its rate of dissipation $\varepsilon$, while near the wall, we conserve the $k$ equation and deduce $\varepsilon$ from $k$ via an algebraic expression. To simplify the notations, we denote $k-\varepsilon$ for the turbulence model in high-Reynolds regions and $k-L$ ( $L$ for length) for the model used for the near-wall treatment. An important point is that the success of turbulence modeling and simulation depends greatly on the treatment of the near-wall regions. This is the reason why the situation remains to be clarified from the mathematical point of view. Another motivation of our work is to decrease the numerical cost for the simulation of flows with the Navier-Stokes equations coupled with the turbulence model with the development of wall-laws [14]. The principle of wall-laws is to remove the stiff-part of the boundary layer, replacing the classical no-slip boundary condition by a more sophisticated relation between the variables and their derivatives. To do so, we find new boundary conditions on a fictitious wall near the real one. This study will validate the development of our wall-laws as, when no experimental data is available, the simulations with the two-layer $k-\varepsilon$ model give us a reference solution. In the past, a new set of variables has been found by Mohammadi [15] which helped to prove that these variables are bounded (and, as a consequence, that $k$ and $\varepsilon$ are bounded too). The change of variables appears if we consider the $k-\varepsilon$ equations and neglect the viscous terms. For incompressible situations, the couple is:

$$
\theta=\frac{k}{\varepsilon} \text { and } \phi=\frac{\varepsilon^{2}}{k^{3}}
$$

With these definitions, Lewandowski and Mohammadi [10] extract from the $k-\varepsilon$ model two new parabolic equations with the 'suitable sign' to demonstrate that the variables exist and are bounded. Several authors have $[1,2,6,7,11,12]$ shown the interest of these new variables. For instance, in [8] and [9], the authors are interested in the coupling of these equations with the Stokes or the Navier-Stokes equations.

Through this paper, we reproduce the change of variables for the $k-L$ model to obtain the $(\theta)$ model. Then, we focus our attention on the singularity of this model in near-wall regions. We present and study an alternative model called $\left(\theta_{\delta}\right)$ in which we have added a correction to remove this singularity. We also study the problem of coupling the Navier-Stokes equations with the $(\theta)$ or $\left(\theta_{\delta}\right)$ model and show that the $\left(\theta_{\delta}\right)$ model is, from the mathematical point of view, more appropriated. Finally, numerical results show the suitability of our new model.

To summarize, the originality of this work concerns the extension to low-Reynolds regions of the previous studies performed for high-Reynolds validity domain $[1,2,6,7,10-12]$.

\section{The Navier-Stokes and turbulence model Equations}

We focus our attention on incompressible turbulent flows. The flow is solution of the Navier-Stokes equations coupled with the turbulence model. In the following, we use $\rho$ for the density, $u$ for the velocity vector and $E$ for the energy. Moreover, let $\nabla u=u_{i, j}$ the gradient of $u, D=u_{i, i}$ its divergence. $P=\left(\nabla u+\nabla u^{t}\right): \nabla u-2 / 3 D^{2}$ represents the production of the turbulence and $S=P: \nabla u$.

The incompressible Navier-Stokes equations are:

$$
\begin{gathered}
D=0 \\
\frac{\partial u}{\partial t}+\nabla \cdot(u \otimes u)+\nabla(p / \rho)=\nabla \cdot\left(\left(\nu+\nu_{t}\right) S\right) \\
\frac{\partial E}{\partial t}+\nabla \cdot((E+p / \rho) u)=\nabla \cdot\left(\left(\nu+\nu_{t}\right) S u\right)+\nabla \cdot\left(\left(\chi+\chi_{t}\right) \nabla T\right)
\end{gathered}
$$

with

$$
\begin{gathered}
\chi=\frac{\gamma \nu}{P r}, \quad \chi_{t}=\frac{\gamma \nu_{t}}{P r_{t}}, \\
\gamma=1.4, \quad \operatorname{Pr}=0.72 \quad \text { et } \quad P r_{t}=0.9 .
\end{gathered}
$$

$\nu$ and $\nu_{t}$ are the laminar and turbulent kinetic viscosities respectively. They are proportional to the inverse of the laminar and turbulent Reynolds numbers. 
To close the previous system, we use a two-layer $k-\varepsilon$ turbulence model. Let $y^{+}$be the local Reynolds number defined by:

$$
y^{+}=\frac{u_{\tau} y}{\nu}
$$

$y$ is the distance between a point $M$ in the flow and the wall boundary $\Gamma_{0} \subset \partial \Omega$; it is defined by:

$$
y=\min \left\{\lambda ; \quad X \vec{M}=\lambda \vec{n} ; \quad X \in \Gamma_{0}, \quad \vec{n} \text { unit normal vector to } \Gamma_{0} \text { at } X\right\} .
$$

$u_{\tau}$ is a constant called the friction velocity. It is evaluated at each point $X$ of the wall boundary such that $\overrightarrow{X P}=\lambda \vec{n}$ :

$$
\left.\nu \frac{\partial u}{\partial y}\right|_{X}=\left|u_{\tau}\right| u_{\tau}
$$

In the high-Reynolds number regions $\left(y^{+}>A\right)$, we have:

$$
\frac{\partial k}{\partial t}+u \nabla k-\nabla\left(\left(\nu+\nu_{t}\right) \nabla k\right)=\nu_{t} P-\varepsilon,
$$

and

$$
\frac{\partial \varepsilon}{\partial t}+u \nabla \varepsilon-\nabla\left(\left(\nu+c_{\varepsilon} \nu_{t}\right) \nabla \varepsilon\right)=c_{1} k P-c_{2} \frac{\varepsilon^{2}}{k} .
$$

This model is closed by the turbulent viscosity:

$$
\nu_{t}=c_{\mu} \frac{k^{2}}{\varepsilon} .
$$

In the low-Reynolds number regions $\left(y^{+} \leq A\right)$, we have:

$$
\frac{\partial k}{\partial t}+u \nabla k-\nabla\left(\left(\nu+\nu_{t}\right) \nabla k\right)=\nu_{t} P-\varepsilon,
$$

and

$$
\varepsilon=\frac{k^{3 / 2}}{l_{\varepsilon}},
$$

with

$$
l_{\varepsilon}=\kappa c_{\mu}^{-3 / 4} y\left(1-\exp \left(\frac{-y^{+}}{2 \kappa c_{\mu}^{-3 / 4}}\right)\right) .
$$

The turbulent viscosity is evaluated with the following relation:

$$
\nu_{t}=c_{\mu} \sqrt{k} l_{\mu}, \text { with } l_{\mu}=\kappa c_{\mu}^{-3 / 4} y\left(1-\exp \left(\frac{-y^{+}}{70}\right)\right) .
$$

The constants $\kappa, c_{\mu}, c_{1}, c_{2}, c_{\varepsilon}$ are respectively equal to $0.41,0.09,0.1296,11 / 6,1 / 1.4245$.

$P$ has a good property: it is always positive. Actually, in $2 \mathrm{D}$, if $u=(U, V)$, we have:

$$
P=\frac{2}{3}\left(U_{, x}-V_{, y}\right)^{2}+\left(U_{, y}+V_{, x}\right)^{2} \geq 0,
$$


and in $3 \mathrm{D}$, if $u=(U, V, W)$, the expression for $P$ is:

$$
\begin{aligned}
P & =\frac{2}{3}\left(\left(U_{, x}-V_{, y}\right)^{2}+\left(U_{, x}-W_{, z}\right)^{2}+\left(V_{, y}-W_{, z}\right)^{2}\right)+\left(U_{, y}+V_{, x}\right)^{2}+\left(U_{, z}+W_{, x}\right)^{2}+\left(V_{, z}+W_{, y}\right)^{2} \\
& \geq 0
\end{aligned}
$$

This aspect of $P$ will be essential along this article.

In the following, we suppose that $\Omega \subset \mathbb{R}^{2}$ or $\mathbb{R}^{3}$ is an open set with a smooth boundary and that the velocity field $u$ is known and regular. To simplify the notations, we define the constants $C_{1}$ and $C_{2}$ :

$$
C_{1}=2 \chi c_{\mu}^{-3 / 4}, C_{2}=70
$$

and, by abuse of notations, let 'stand for $\mathrm{d} / \mathrm{d} t$.

Remark 1.1. The choice of $A$ defining the application domains of the $k-\varepsilon$ and $k-L$ turbulence models is unknown a priori. For the physicists, $A>70$. However, if $A=200$, we have $l_{\mu} \simeq l_{\varepsilon} \simeq \kappa c_{\mu}^{-3 / 4} y$. In particular, this will be useful for the consistency between both models at the interface $y^{+}=200$ (see next section).

\section{Consistency of the $k-\varepsilon$ And $k-L$ Models - Link AT The interface $y^{+}=200$}

As we have seen in the previous section, $y^{+}$is the local Reynolds number. To prove the consistency of the models, we need to demonstrate the continuity of $k$ and $\nu_{t}$ at the interface.

Because of the values of the constants, one easily sees that, at $y^{+}=200$, the $k-\varepsilon$ model leads to:

$$
\frac{\partial k}{\partial t}+u \nabla k-\nabla \cdot\left(\left(\nu+\nu_{t}\right) \nabla k\right)=c_{\mu}^{0.25} \sqrt{k} y P-\frac{k^{3 / 2}}{l_{\varepsilon}},
$$

with

$$
\nu_{t}=\frac{k^{2}}{\varepsilon}=\kappa c_{\mu}^{-3 / 4} \sqrt{k} y
$$

as, at $y^{+}=200$, the boundary condition for $\varepsilon$ is a Dirichlet one, and comes from the $k-L$ model.

For the $k-L$ turbulence model, the equation in $k$ is the same:

$$
\frac{\partial k}{\partial t}+u \nabla k-\nabla \cdot\left(\left(\nu+\nu_{t}\right) \nabla k\right)=c_{\mu}^{0.25} \sqrt{k} y P-\frac{k^{3 / 2}}{l_{\varepsilon}},
$$

and $\nu_{t}$ has the same expression:

$$
\nu_{t}=\kappa c_{\mu}^{-3 / 4} \sqrt{k} y
$$

So, we have shown that the models are coherent at the interface $y^{+}=200$ at the 0 order (continuity).

\section{3. $\theta$ EQUATION EXTRACTED FROM THE $k-L$ MODEL}

In [15] has been proposed a new pair of variables $\theta-\phi$ for the high Reynolds number regions $\left(y^{+}>200\right)$ :

$$
\theta=\frac{k}{\varepsilon} \text { et } \phi=\frac{\varepsilon^{2}}{k^{3}}
$$

Here, this leads to:

$$
\theta=\frac{l_{\varepsilon}}{\sqrt{k}} \text { et } \phi=\frac{1}{l_{\varepsilon}^{2}} .
$$


The variable $\theta$ is interesting. What is the boundary condition for $\theta$ ? The physicists suppose that, at the wall, $\varepsilon=C_{s t}>0$ and $k=0$; therefore:

at the wall.

$$
\theta_{\Gamma_{0}}=\lim _{y \rightarrow 0} \frac{k}{\varepsilon}=\frac{0}{C_{s t}}=0
$$

Let us find the PDE for $\theta$. We remark that:

$$
\frac{\partial \theta}{\partial t}=-\frac{1}{2} \frac{1}{k^{3 / 2} l_{\varepsilon}} \frac{\partial k}{\partial t}
$$

As a consequence

$$
\theta^{\prime}+u \cdot \nabla \theta-\operatorname{Diff}_{\theta}=-c_{\mu} \frac{l_{\mu}}{l_{\varepsilon}} P \theta^{2}+\frac{1}{2}
$$

where $\operatorname{Diff}_{\theta}$ contains the term coming from the viscous one in the $k$ equation. We suppose, as in [10], that it is responsible for the diffusion of $\theta$ and is modeled by:

$$
\operatorname{Diff}_{\theta}=\nabla \cdot\left(\left(\nu+c_{\theta} \nu_{t}\right) \nabla \theta\right)
$$

where $c_{\theta}$ can be a positive function of $y^{+}$or a new non-negative constant. To simplify the expressions, we will suppose that $c_{\theta}$ is a strictly positive constant, but this point is not crucial and the same result can be obtained with a positive function.

Finally, the $\theta$ equation is:

$$
\theta^{\prime}+u \cdot \nabla \theta-\nabla \cdot\left(\left(\nu+c_{\theta} \nu_{t}\right) \nabla \theta\right)=-c_{\mu} \frac{l_{\mu}}{l_{\varepsilon}} P \theta^{2}+\frac{1}{2}
$$

with $\nu_{t}=c_{\mu} l_{\mu} l_{\varepsilon} / \theta$. Moreover, the initial and boundary conditions are:

$$
\theta(x, 0)=\theta_{0} \geq 0, \theta=0 \text { on } \Gamma_{0} \text { and } \nabla \theta \cdot \vec{n}=0 \text { on } \Gamma_{1},
$$

where $\Gamma_{1} \cap \Gamma_{0}=\emptyset, \Gamma_{0} \cup \Gamma_{1}=\partial \Omega$ and $\vec{n}$ is an inward vector normal to $\Gamma_{1}$ in each point of the boundary. To simplify the notations, let $\nabla \theta_{\Gamma_{1}}=0$ stand for $\nabla \theta \cdot \vec{n}=0$ on $\Gamma_{1}$.

\section{A MOdel PROBLEM}

We consider the following problem

$$
\left\{\begin{array}{l}
\theta^{\prime}+u \cdot \nabla \theta-\nu \triangle \theta=-c_{\mu} \frac{l_{\mu}}{l_{\varepsilon}} P \theta^{2}+\frac{1}{2} \\
\theta(t=0)=\theta_{0} \geq 0 \\
\theta_{\partial \Omega}=0
\end{array}\right.
$$

that comes from (14) (we suppose $\nu+c_{\theta} \nu_{t}=C_{s t}$ ).

In reality, we will study the new problem that follows:

$$
\left\{\begin{array}{l}
\theta^{\prime}+u \cdot \nabla \theta-\nu \triangle \theta=-c_{\mu} \frac{l_{\mu}}{l_{\varepsilon}} P \theta|\theta|+\frac{1}{2} \\
\theta(t=0)=\theta_{0} \geq 0 \\
\theta_{\partial \Omega}=0 .
\end{array}\right.
$$

As we will see, the solution of (16) is positive and will be the solution of the system (15). 
Equation (16) must be understood in the distribution sense and this will enable us to deduce the spaces for $\theta, u$ and $P$. Let $\Phi \in \mathcal{C}_{c}^{\infty}([0, T), \Omega)=\mathcal{D}([0, T) \times \Omega)$, we have:

$$
\begin{aligned}
-\int_{0}^{T} \mathrm{~d} t \int_{\Omega} \mathrm{d} x \frac{\partial \Phi}{\partial t} \theta-\int_{\Omega} \mathrm{d} x \theta \Phi(0, x)-\int_{0}^{T} \mathrm{~d} t \int_{\Omega} \mathrm{d} x \theta u \nabla \Phi+\nu \int_{0}^{T} \mathrm{~d} t \int_{\Omega} \mathrm{d} x \nabla \theta \nabla \Phi= \\
-c_{\mu} \int_{0}^{T} \mathrm{~d} t \int_{\Omega} \frac{l_{\mu}}{l_{\varepsilon}} P \theta|\theta| \Phi+\frac{1}{2} \int_{0}^{T} \mathrm{~d} t \int_{\Omega} \mathrm{d} x \Phi
\end{aligned}
$$

As a consequence, relation (17) is meaningful if:

$$
\theta \in L^{\infty}\left([0, T), H_{0}^{1}(\Omega)\right), u \in L^{2}\left([0, T), L^{\infty}(\Omega)\right), \text { et } P \in L^{2}\left([0, T), L^{\infty}(\Omega)\right) .
$$

As a matter of fact, the convection term leads to:

$$
\int_{0}^{T} \mathrm{~d} t \int_{\Omega} \mathrm{d} x \theta u \nabla \Phi \leq\|\nabla \Phi\|_{\infty} \int_{0}^{T} \mathrm{~d} t \int_{\Omega} \mathrm{d} x|\theta u| .
$$

For the nonlinear term, we deduce:

$$
\int_{0}^{T} \mathrm{~d} t \int_{\Omega} \mathrm{d} x \frac{l_{\mu}}{l_{\varepsilon}} \theta|\theta| \Phi P \leq C\|\Phi\|_{\infty} \int_{0}^{T}\|P\|_{\infty} \int_{\Omega} \mathrm{d} x|\theta|^{2},
$$

with $C=\max \left(l_{\mu} / l_{\varepsilon}\right)=C_{1} / C_{2}\left(C_{1}\right.$ and $C_{2}$ defined by $\left.(9)\right)$.

Theorem 4.1. Let $u \in L^{\infty}\left([0, T), L^{\infty}(\Omega)\right)$, and $P \in L^{\infty}\left([0, T), L^{\infty}(\Omega)\right)$, and suppose $\theta_{0} \in L^{\infty}(\Omega) \cap H_{0}^{1}(\Omega)$, with $\theta_{0} \geq 0$. Then, the problem (16) has a unique solution $\theta$ such that:

$$
\theta \in L^{\infty}\left([0, T), L^{\infty}(\Omega)\right) \cap L^{2}\left([0, T) ; H_{0}^{1}(\Omega)\right)
$$

and

$$
\theta \geq 0 \text { in } \Omega \times[0, T)
$$

Proof. The proof, which is done in several steps, is based on a compactness method [13, 18]. First, we find a priori estimates from the equation (Lems. 4.2, 4.3 and 4.4). Then, we prove the existence of a solution of the problem thanks to the Faedo-Galerkin method (Sect. 4.2). For physical reasons, we need to demonstrate that the solution is positive (Lem. 4.7). Finally, in Lemma 4.8, we prove the uniqueness of the solution.

\subsection{A priori estimates for $\boldsymbol{\theta}$}

Lemma 4.2. If $\theta_{0} \in H_{0}^{1}(\Omega)$ and if $u \in L^{2}\left([0, T) ; L^{\infty}(\Omega)\right)$ and $P \in L^{\infty}\left([0, T) ; L^{\infty}(\Omega)\right)$, then $\theta \in L^{\infty}([0, T)$; $\left.L^{2}(\Omega)\right) \cap L^{2}\left([0, T) ; H_{0}^{1}(\Omega)\right)$.

Proof. Let us work in $H_{0}^{1}(\Omega)$. Multiplying equation (16) by $\theta$ and integrating in space leads to:

$$
\frac{1}{2} \frac{\mathrm{d}}{\mathrm{d} t} \int_{\Omega}|\theta|^{2}+\nu \int_{\Omega}|\nabla \theta|^{2}=-c_{\mu} \int_{\Omega} \frac{l_{\mu}}{l_{\varepsilon}} P|\theta|^{3}+\frac{1}{2} \int_{\Omega} \theta-\int_{\Omega} \theta u \cdot \nabla \theta .
$$

As $P$ is positive and as $1 \leq l_{\mu} / l_{\varepsilon} \leq C_{1} / C_{2}$, (18) becomes, using Young inequality:

$$
\frac{1}{2} \frac{\mathrm{d}}{\mathrm{d} t} \int_{\Omega}|\theta|^{2} \leq \frac{1}{4} \operatorname{mes}(\Omega)+\frac{1}{4} \int_{\Omega}|\theta|^{2}+\left|\int_{\Omega} \theta u \cdot \nabla \theta\right|
$$


When integrated by parts, the last term of relation (19) becomes:

$$
\int_{\Omega} \theta u \cdot \nabla \theta=-\frac{1}{2} \int_{\Omega} \mathrm{D} \theta^{2}=0
$$

And relation (19) leads to:

$$
\frac{1}{2} \frac{\mathrm{d}}{\mathrm{d} t} \int_{\Omega}|\theta|^{2} \leq C \int_{\Omega}|\theta|^{2}+C^{\prime}
$$

with $C=1 / 4$ and $C^{\prime}=\operatorname{mes}(\Omega) / 4$. From Gronwall's lemma, $(21)$ gives:

$$
\frac{1}{2} \int_{\Omega}|\theta|^{2} \leq e^{2 C T} \int_{\Omega}\left|\theta_{0}\right|^{2}+C^{\prime \prime}
$$

where $C^{\prime \prime}=C^{\prime} / C\left(e^{2 C T}-1\right)$. As a consequence, we have:

$$
\theta \in L^{\infty}\left([0, T) ; L^{2}(\Omega)\right)
$$

Moreover, integrating relation (18) between 0 and $T$ and still using the positivity of $P$, one obtains:

$$
\nabla \theta \in L^{2}\left([0, T) ; L^{2}(\Omega)\right) .
$$

And so

$$
\theta \in L^{2}\left([0, T) ; H_{0}^{1}(\Omega)\right) \cap L^{\infty}\left([0, T) ; L^{2}(\Omega)\right)
$$

Lemma 4.3. If we have the same hypotheses as in Lemma 4.2 and if we also suppose $\theta_{0} \in L^{p}(\Omega)$ for $1 \leq p<\infty$, then:

$$
\theta \in L^{2}\left([0, T) ; H_{0}^{1}(\Omega)\right) \cap L^{\infty}\left([0, T) ; L^{\infty}(\Omega)\right) .
$$

Proof. The proof is the same as for Lemma 4.2. We multiply equation (16) by $|\theta|^{p-2} \theta$ and use the previous technique and the fact that $P \geq 0$ which is crucial.

Lemma 4.4. Under the hypotheses of Lemma 4.3, $\theta^{\prime} \in L^{2}\left([0, T) ; L^{2}(\Omega)\right)$.

Proof. Let us multiply formally equation (16) by $\theta^{\prime}$ and integrate in space and time:

$$
\begin{gathered}
\int_{0}^{T} \mathrm{~d} t \int_{\Omega} \mathrm{d} x\left|\theta^{\prime}\right|^{2}+\int_{0}^{T} \mathrm{~d} t \int_{\Omega} \mathrm{d} x u \nabla \theta \theta^{\prime}+\nu \int_{0}^{T} \mathrm{~d} t \int_{\Omega} \mathrm{d} x \nabla \theta \nabla\left(\theta^{\prime}\right)= \\
-c_{\mu} \int_{0}^{T} \mathrm{~d} t \int_{\Omega} \mathrm{d} x \frac{l_{\mu}}{l_{\varepsilon}} P \theta^{2} \theta^{\prime}+\frac{1}{2} \int_{0}^{T} \mathrm{~d} t \int_{\Omega} \mathrm{d} x \theta^{\prime}
\end{gathered}
$$

We have:

$$
\nu \int_{0}^{T} \mathrm{~d} t \int_{\Omega} \mathrm{d} x \nabla \theta \nabla\left(\theta^{\prime}\right)=\frac{\nu}{2} \int_{0}^{T} \mathrm{~d} t \int_{\Omega} \mathrm{d} x \frac{\mathrm{d}}{\mathrm{d} t}\left(|\nabla \theta|^{2}\right)=\frac{\nu}{2} \int_{\Omega} \mathrm{d} x|\nabla \theta(T, .)|^{2}-\frac{\nu}{2} \int_{\Omega} \mathrm{d} x\left|\nabla \theta_{0}\right|^{2}
$$

because $\nabla \theta \in L^{2}\left([0, T) ; L^{2}(\Omega)\right)$ and $\theta_{0} \in L^{\infty}(\Omega) \cap H_{0}^{1}(\Omega)$.

It is easy to prove that:

$$
\left|\int_{0}^{T} \mathrm{~d} t \int_{\Omega} \mathrm{d} x u \nabla \theta \theta^{\prime}\right| \leq\|u\|_{L^{\infty}\left([0, T), L^{\infty}(\Omega)\right)}\|\nabla \theta\|_{L^{2}\left([0, T), L^{2}(\Omega)\right)}\left\|\theta^{\prime}\right\|_{L^{2}\left([0, T), L^{2}(\Omega)\right)},
$$


and

$$
\left|\int_{0}^{T} \mathrm{~d} t \int_{\Omega} \mathrm{d} x \frac{l_{\mu}}{l_{\varepsilon}} P \theta\right| \theta\left|\theta^{\prime}\right| \leq \frac{C_{1}}{C_{2}}\|P\|_{L^{2}\left([0, T), L^{\infty}(\Omega)\right)}\|\theta\|_{L^{\infty}\left([0, T), L^{\infty}(\Omega)\right)}^{2}\left\|\theta^{\prime}\right\|_{L^{2}\left([0, T), L^{2}(\Omega)\right)} .
$$

Using these relations in (23), one obtains:

$$
\left\|\theta^{\prime}\right\|_{L^{2}\left([0, T) ; L^{2}(\Omega)\right)}^{2} \leq C\left\|\theta^{\prime}\right\|_{L^{2}\left([0, T) ; L^{2}(\Omega)\right.}+C^{\prime}
$$

where $C$ and $C^{\prime}$ are two non-negative constants independent of $\theta$. And so:

$$
\theta^{\prime} \in L^{2}\left([0, T) ; L^{2}(\Omega)\right)
$$

\subsection{Existence}

Lemma 4.5. Under the hypotheses of Theorem $4.1\left(u \in L^{\infty}\left([0, T) ; L^{\infty}(\Omega)\right), P \in L^{\infty}\left([0, T) ; L^{\infty}(\Omega)\right)\right.$ and $\theta_{0} \in$ $\left.L^{\infty}(\Omega) \cap H_{0}^{1}(\Omega)\right)$, the problem (16) has a solution.

Proof. Consider the bilinear form $a$ defined by:

$$
\begin{aligned}
a: H_{0}^{1}(\Omega) \times H_{0}^{1}(\Omega) & \longrightarrow \mathbb{R} \\
(u, v) & \longrightarrow \nu \int_{\Omega} \nabla u \nabla v .
\end{aligned}
$$

$a$ is a symmetric, strictly positive, coercive self-adjoint bilinear continuous operator, and a linear application $A$ can be associated to $a$ by:

$$
\begin{aligned}
A: H_{0}^{1}(\Omega) & \longrightarrow H_{0}^{1}(\Omega) \\
u & \longrightarrow A(u)(.)=a(u, .) .
\end{aligned}
$$

Let $\left(\lambda_{i}\right)_{i \in \mathbb{N}}$, be the family of eigenvalues of $A$ (in increasing order) and the family $\left(w_{i}\right)_{i \in \mathbb{N}}$ constituted of eigenvectors $w_{i}$ of $A$ associated with the eigenvalues $\lambda_{i}$ respectively, which is orthonormal in $L^{2}(\Omega)$ and orthogonal for $a$. We have:

$$
\begin{gathered}
a\left(w_{i}, w_{j}\right)=\lambda_{i} \delta_{i j}, \quad \forall i, j \in \mathbb{N}, \\
\int_{\Omega} w_{i} w_{j}=\delta_{i j}, \quad \forall i, j \in \mathbb{N},
\end{gathered}
$$

where $\delta$ is the Kronecker symbol.

We consider the linear subspaces $V_{m}=\operatorname{vect}\left\{w_{1}, \ldots, w_{m}\right\}$ for $m \geq 1$ and let $\theta_{0 m}$ be the projection of $\theta_{0}$ on $V_{m}$; we look for $\theta_{m}$ of the form:

$$
\theta_{m}=\sum_{i=1}^{m} g_{i, m}(t) w_{i}
$$

and solution of the following problem:

$$
\left\{\begin{array}{l}
\int_{\Omega} \theta_{m}^{\prime} w_{i}+\int_{\Omega} u \nabla \theta_{m} w_{i}+a\left(\theta_{m}, w_{i}\right)=-c_{\mu} \int_{\Omega} \frac{l_{\mu}}{l_{\varepsilon}} P\left|\theta_{m}\right| \theta_{m} w_{i}+\frac{1}{2} \int_{\Omega} w_{i} . \\
\theta_{m}(0, .)=\theta_{0 m}(.)
\end{array}\right.
$$


As a consequence, we obtain a differential system in finite dimension and the classical theorems of differential equations can be applied to obtain the existence of $\theta_{m}$ with a form like $(25)$ and defined on an interval $\left[0, T_{m}\right)$. The a priori estimates that can already be used in finite dimension (because $\left\|\theta_{0 m}\right\|_{L^{2}(\Omega)} \leq\left\|\theta_{0}\right\|_{L^{2}(\Omega)}$ ), ensure that, for all $m$, the solution $\theta_{m}$ is defined on the same interval $[0, T)$.

We have found $\theta_{m}$ such that $\theta_{m} \in L^{\infty}\left([0, T) ; L^{\infty}(\Omega)\right) \cap L^{2}\left([0, T) ; H_{0}^{1}(\Omega)\right)$ and $\theta_{m}^{\prime} \in L^{2}\left([0, T) ; L^{2}(\Omega)\right)$. Moreover, the bounds that ensure $\theta_{m}$ to be in these spaces are independent of $m$ and we can extract a subsequence of $\theta_{m}$ (still written $\left.\theta_{m}\right)$ and there exists $\theta$, with $\theta \in L^{\infty}\left([0, T) ; L^{\infty}(\Omega)\right) \cap L^{2}\left([0, T) ; H_{0}^{1}(\Omega)\right)$ and $\theta^{\prime} \in L^{2}\left([0, T) ; L^{2}(\Omega)\right)$, such that:

- $\theta_{m} \longrightarrow \theta$ weakly in $H^{1}\left([0, T) ; L^{2}(\Omega)\right)$,

- $\nabla \theta_{m} \longrightarrow \nabla \theta$ weakly in $L^{2}\left([0, T) ; L^{2}(\Omega)\right)$.

We obtain readily:

$$
\int_{0}^{T} \int_{\Omega} \theta_{m}^{\prime} \Phi \longrightarrow \int_{0}^{T} \int_{\Omega} \theta^{\prime} \Phi \text { and } \int_{0}^{T} \int_{\Omega} \nabla \theta_{m} \nabla \Phi \longrightarrow \int_{0}^{T} \int_{\Omega} \nabla \theta \nabla \Phi, \quad \forall \Phi \in \mathcal{D}([0, T) \times \Omega) .
$$

We have to demonstrate the following convergences:

$$
\begin{gathered}
\int_{0}^{T} \int_{\Omega} u \nabla \theta_{m} \Phi \longrightarrow \int_{0}^{T} \int_{\Omega} u \nabla \theta \Phi, \quad \forall \Phi \in \mathcal{D}([0, T) \times \Omega) \\
\int_{0}^{T} \int_{\Omega} \frac{l_{\mu}}{l_{\varepsilon}} P\left|\theta_{m}\right| \theta_{m} \Phi \longrightarrow \int_{0}^{T} \int_{\Omega} \frac{l_{\mu}}{l_{\varepsilon}} P|\theta| \theta \Phi \quad \forall \Phi \in \mathcal{D}([0, T) \times \Omega) .
\end{gathered}
$$

The weak convergence in $H^{1}\left([0, T) ; L^{2}(\Omega)\right)$ implies the strong one in $L^{2}\left([0, T) ; L^{2}(\Omega)\right)$ and therefore:

- $\theta_{m} \longrightarrow \theta$ strongly in $L^{2}\left([0, T) ; L^{2}(\Omega)\right)$;

- $\theta_{m} \longrightarrow \theta$, a.e. $x \in \Omega$, a.e. $t \in[0, T)$.

From the strong convergence of $\theta_{m}$, we deduce the convergence $(26)$ : if $\Phi \in \mathcal{D}([0, T) \times \Omega)$, writing $\nabla \cdot(u \Phi)=$ $u \nabla \Phi+D \Phi=u \nabla \Phi$, one sees that, thanks to the hypotheses on $u$, the convergence (26) occurs. Let us verify convergence $(27)$.

$\theta_{m}$ is in a bounded set of $L^{\infty}\left([0, T) ; L^{\infty}(\Omega)\right)$ and the bounds are independent of $m$. In particular, $\left|\theta_{m}\right| \theta_{m}$ is in a bounded set of $L^{2}\left([0, T) ; L^{2}(\Omega)\right)$, with the bounds independent of $m$. As $\theta_{m}$ converges to $\theta$ for a.e. $t$, $x$, we also have the convergence of $\theta_{m}\left|\theta_{m}\right|$ to $\theta|\theta|$ for a.e. $t, x$. As a consequence, thanks to the following lemma (see Chap. 1 in [13]), $\theta_{m}\left|\theta_{m}\right| \longrightarrow \theta|\theta|$ weakly in $L^{2}\left([0, T) ; L^{2}(\Omega)\right)$.

Lemma 4.6. Let $q, n \in \mathbb{N}$ such that $1<q<\infty$. Let $\mathcal{O}$ be an open set of $\mathbb{R}^{n} \times \mathbb{R}, g_{n}$ a sequence of functions in $L^{q}(\mathcal{O})$ and $g \in L^{q}(\mathcal{O})$ such that $\left\|g_{n}\right\|_{L^{q}(\mathcal{O})} \leq C$ and $g_{n} \longrightarrow g$ a.e. in $\mathcal{O}$. Then, $g_{n} \longrightarrow g$ weakly in $L^{q}(\mathcal{O})$. 
So, if $\Phi$ is a test function, we have:

$$
\begin{aligned}
\left|\int_{0}^{T} \int_{\Omega} \frac{l_{\mu}}{l_{\varepsilon}} P\left(\left|\theta_{m}\right| \theta_{m}-|\theta| \theta\right) \Phi\right| & \leq C \int_{0}^{T} \int_{\Omega}|P|\left|\left(\left|\theta_{m}\right| \theta_{m}-|\theta| \theta\right) \Phi\right| \\
& \leq C\|P\|_{L^{\infty}\left([0, T) ; L^{\infty}(\Omega)\right)} \int_{0}^{T} \int_{\Omega}\left|\left(\left|\theta_{m}\right| \theta_{m}-|\theta| \theta\right) \Phi\right|
\end{aligned}
$$

and convergence (27) occurs.

The initial condition has still to be verified for the demonstration to be complete: do we have $\theta(0, x)=\theta_{0}(x)$ a.e. $x \in \Omega$ ? The answer is clearly yes. Let $\phi$ be a function in $\mathcal{C}^{\infty}([0, T))$ such that $\phi(0) \neq 0$ and $\phi=0$ in a neighborhood of $T$. Let $v$ in $H_{0}^{1}(\Omega)$. As a consequence, $\psi=\phi \otimes v \in W\left(0, T ; H_{0}^{1}(\Omega), H^{-1}(\Omega)\right)$ (see [4] par. 1 and 3 ) where $\psi(t, x)=\phi(t) v(x)$. So

$$
\begin{aligned}
\int_{0}^{T} \int_{\Omega} \theta^{\prime} \psi & =\int_{0}^{T} \int_{\Omega} \theta^{\prime}(t, x) \phi(t) v(x) \mathrm{d} x \mathrm{~d} t \\
& =-\int_{0}^{T} \int_{\Omega} \theta(t, x) \phi^{\prime}(t) v(x) \mathrm{d} x \mathrm{~d} t-\int_{\Omega} \theta(0, x) v(x) \phi(0) .
\end{aligned}
$$

Moreover, if $v_{m}$ is the projection of $v$ on $V_{m}$, we deduce from $\theta_{m}$ :

$$
\begin{aligned}
\int_{0}^{T} \int_{\Omega} \theta_{m}^{\prime} \psi & =\int_{0}^{T} \int_{\Omega} \theta_{m}^{\prime}(t, x) \phi(t) v(x) \mathrm{d} x \mathrm{~d} t \\
& =-\int_{0}^{T} \int_{\Omega} \theta_{m}(t, x) \phi^{\prime}(t) v_{m}(x) \mathrm{d} x \mathrm{~d} t-\int_{\Omega} \theta_{m}(0, x) v_{m}(x) \phi(0) \\
& =-\int_{0}^{T} \int_{\Omega} \theta_{m}(t, x) \phi^{\prime}(t) v_{m}(x) \mathrm{d} x \mathrm{~d} t-\int_{\Omega} \theta_{0 m}(x) v_{m}(x) \phi(0)
\end{aligned}
$$

$\theta_{0 m}(x)=\theta_{m}(0, x)$. When $m$ goes to $\infty$, we deduce from the previous relations:

$$
\int_{\Omega} \theta_{0}(x) v(x)=\int_{\Omega} \theta(0, x) v(x), \forall v \in H_{0}^{1}(\Omega)
$$

Therefore, from the compact injection of $H_{0}^{1}(\Omega)$ in $L^{2}(\Omega)$, relation $(28)$ is still valid for all $v \in L^{2}(\Omega)$ and, as a consequence,

$$
\theta_{0}(x)=\theta(0, x) \text { a.e. } x \in \Omega .
$$

As a conclusion, $\theta$ is a solution of problem (15).

\subsection{Positivity}

Lemma 4.7. Under the hypotheses of Theorem 4.1, if $\theta_{0} \geq 0$, then $\theta \geq 0$ a.e. $t$ and $x$.

Proof. We write $\theta=\theta^{+}-\theta^{-}$where $\theta^{+}$and $\theta^{-}$are positive. Multiplying the equation in $\theta$ of (16) by $-\theta^{-}$and using a relation like $(20)$, we have:

$$
\frac{1}{2} \frac{\mathrm{d}}{\mathrm{d} t} \int_{\Omega}\left|\theta^{-}\right|^{2}+\nu \int_{\Omega}\left|\nabla \theta^{-}\right|^{2}=-c_{\mu} \int_{\Omega} \frac{l_{\mu}}{l_{\varepsilon}} P\left|\theta^{-}\right|^{3}-\frac{1}{2} \int_{\Omega} \theta^{-} .
$$

Due to the positivity of $P, l_{\mu}, l_{\varepsilon}$ and the fact that $\theta^{-} \geq 0,(29)$ becomes:

$$
\frac{1}{2} \frac{\mathrm{d}}{\mathrm{d} t} \int_{\Omega}\left|\theta^{-}\right|^{2} \leq 0
$$


from which we deduce

$$
\theta^{-}=0 \text { a.e. } t, x
$$

as $\theta_{0}^{-}=0$

\subsection{Uniqueness of $\theta$}

Lemma 4.8. Under the hypotheses of Theorem 4.1, there exists only one solution to problem (16).

Proof. Let $\theta_{1}$ and $\theta_{2}$ be two solutions of the problem (16). Let $\Phi=\theta_{1}-\theta_{2}$. $\Phi$ is solution of the following problem:

$$
\left\{\begin{array}{l}
\Phi^{\prime}+u \cdot \nabla \Phi-\nu \triangle \Phi=-c_{\mu} \frac{l_{\mu}}{l_{\varepsilon}} P \Phi\left(\theta_{1}+\theta_{2}\right) \\
\Phi(t=0)=\Phi_{0}=0 \\
\Phi_{\partial \Omega}=0 .
\end{array}\right.
$$

Multiply (31) by $\Phi$ and integrate in space. We find, as $\theta_{1}$ and $\theta_{2}$ are positive and because of the hypotheses on $P$ and $D$ :

$$
\frac{\mathrm{d}}{\mathrm{d} t} \int_{\Omega} \Phi^{2} \leq 0
$$

As $\Phi_{0}=0$, we deduce from (32):

$$
\int_{\Omega} \Phi^{2} \leq 0, \forall t \in[0, T)
$$

and $\theta_{1}=\theta_{2}$ a.e. $x, t$

As a consequence, all the sequence $\left(\theta_{n}\right)_{n \in \mathbb{N}}$ converges to $\theta$.

\section{The initial PRoblem}

Now, we will solve the initial problem in the physical context of incompressible flows. The problem we consider is the following:

$$
\left\{\begin{array}{l}
\theta^{\prime}+u \cdot \nabla \theta-\nabla \cdot\left(\left(\nu+c_{\theta} \frac{c_{\mu} l_{\mu} l_{\varepsilon}}{\theta}\right) \nabla \theta\right)=-c_{\mu} \frac{l_{\mu}}{l_{\varepsilon}} P \theta|\theta|+\frac{1}{2} \\
\theta(x, 0)=\theta_{0} \geq 0 \\
\nabla \theta_{\Gamma_{1}}=0 \\
\theta_{\Gamma_{0}}=0 .
\end{array}\right.
$$

From now on, let $H(\Omega)$ be the space:

$$
H(\Omega)=\left\{u \in H^{2}(\Omega), \quad u=0 \text { on } \Gamma_{0} \text { and } \nabla u \cdot \vec{n}=0 \text { on } \Gamma_{1}\right\}
$$

Theorem 5.1. Let $\left.\Omega=\prod_{i=1}^{3}\right] 0, a_{i}\left[, a_{i}>0\right.$ for $i=1,3$ and $\left.\Gamma_{0}=\right] 0, a_{1}[\times\{0\} \times] 0, a_{3}[\subset \partial \Omega$ the wall boundary with $\vec{n}$ the inward unit vector normal to $\Gamma_{0}$. Let $u \in L^{\infty}\left([0, T) ; L^{\infty}(\Omega)\right), P \in L^{\infty}\left([0, T) ; L^{\infty}(\Omega)\right)$ and $\theta_{0} \in$ $H(\Omega) \cap L^{\infty}(\Omega)$. We suppose that there exists a constant $C$ such that $\theta_{0} \geq C y^{2} \geq 0$ and:

$$
C c_{\mu} \frac{C_{1}}{C_{2}} M_{1} M_{2} b^{2}-\frac{1}{2}<0
$$

where $M_{1}=\|P\|_{L^{\infty}\left([0, T) ; L^{\infty}(\Omega)\right)}, M_{2}$ the uniform upper bound of $\theta_{\delta}$ in $L^{\infty}\left([0, T) ; L^{\infty}(\Omega)\right)$, and $b$ an upper bound of the distance $y$ (defined in (3)). 
Then, there exists a solution $\theta$ of problem $(\mathcal{P})$ and $\theta$ is such that:

$$
\theta \in L^{\infty}\left([0, T) ; L^{\infty}(\Omega)\right) \cap L^{2}([0, T) ; H(\Omega)), \theta^{\prime} \in L^{2}\left([0, T) ; H^{-1}(\Omega)\right)
$$

and

$$
\theta \geq C y^{2} \geq 0
$$

Proof. We will approach the problem $(\mathcal{P})$ by a sequence of problems that will have a solution. Then, we will show that the solutions of the alternative problems converge to a solution of problem $(\mathcal{P})$.

\subsection{A sequence of alternative problems}

We define the alternative problems $\left(\mathcal{P}_{\delta}\right)$ by:

$$
\left\{\begin{array}{l}
\theta_{\delta}^{\prime}+u \cdot \nabla \theta_{\delta}-\nabla \cdot\left(\left(\nu+c_{\theta} \frac{l_{\mu} l_{\varepsilon}}{\sqrt{\theta_{\delta}^{2}+\delta^{2}}}\right) \nabla \theta_{\delta}\right)=-c_{\mu} \frac{l_{\mu}}{l_{\varepsilon}} P \theta_{\delta}\left|\theta_{\delta}\right|+\frac{1}{2}, \\
\theta_{\delta}(x, 0)=\theta_{0} \geq 0 \\
\nabla \theta_{\delta \Gamma_{1}}=0 \\
\theta_{\delta \Gamma_{0}}=0 .
\end{array}\right.
$$

The problem $\left(\mathcal{P}_{\delta}\right)$ has a solution with a certain regularity and which is positive.

\subsection{A priori estimates}

We apply the same method as in the constant eddy viscosity case. The difference comes from the term $u \cdot \nabla \theta_{\delta}$, which we have to account for. Multiply equation (34) by $\theta_{\delta}$ and integrate in space; we have:

$$
\frac{1}{2} \frac{\mathrm{d}}{\mathrm{d} t} \int_{\Omega} \theta_{\delta}^{2}+\int_{\Omega} u \cdot \nabla \theta_{\delta} \theta_{\delta}+\int_{\Omega}\left(\nu+c_{\theta} \frac{c_{\mu} l_{\mu} l_{\varepsilon}}{\sqrt{\theta_{\delta}^{2}+\delta^{2}}}\right) \nabla \theta_{\delta}^{2}=-c_{\mu} \int_{\Omega} \frac{l_{\mu}}{l_{\varepsilon}} P\left|\theta_{\delta}\right|^{3}+\frac{1}{2} \int_{\Omega} \theta_{\delta} .
$$

But, we have:

and using Young's inequality,

$$
\left|\int_{\Omega} u \cdot \nabla \theta_{\delta} \theta_{\delta}\right| \leq\|u\|_{L^{\infty}\left([0, T) ; L^{\infty}(\Omega)\right)} \int_{\Omega}\left|\nabla \theta_{\delta}\right|\left|\theta_{\delta}\right|,
$$

$$
\left|\int_{\Omega} u \cdot \nabla \theta_{\delta} \theta_{\delta}\right| \leq M\left(\alpha \int_{\Omega}|\nabla \theta|^{2}+\frac{1}{\alpha} \int_{\Omega}\left|\theta_{\delta}\right|^{2}\right)
$$

where $\alpha$ is a non negative constant that will be specified below and $M=\|u\|_{L^{\infty}\left([0, T) ; L^{\infty}(\Omega)\right)}$. So, relation (35) leads to:

$$
\frac{1}{2} \frac{\mathrm{d}}{\mathrm{d} t} \int_{\Omega} \theta_{\delta}^{2}+\int_{\Omega}(\nu-\alpha M) \nabla \theta_{\delta}^{2} \leq \frac{M}{\alpha} \int_{\Omega}\left|\theta_{\delta}\right|^{2}+\frac{1}{2} \int_{\Omega} \theta_{\delta} .
$$

If $\alpha$ is such that $\nu-\alpha M>0$, we have:

$$
\frac{1}{2} \frac{\mathrm{d}}{\mathrm{d} t} \int_{\Omega} \theta_{\delta}^{2} \leq C \int_{\Omega} \theta_{\delta}^{2}+C^{\prime}
$$

with $C$ and $C^{\prime}$ independent of $\delta$ and using Gronwall's lemma, we have

$$
\theta_{\delta} \in L^{2}\left([0, T) ; L^{2}(\Omega)\right) .
$$


Moreover, integrating relation (36) between 0 and $T$, we show that:

$$
\nabla \theta_{\delta} \in L^{2}\left([0, T) ; L^{2}(\Omega)\right)
$$

Multiplying the equation by $\left|\theta_{\delta}\right|^{p-2} \theta_{\delta}$ shows that $\theta_{\delta} \in L^{\infty}\left([0, T) ; L^{\infty}(\Omega)\right)$. As a consequence

$$
\theta_{\delta} \in L^{2}([0, T) ; H(\Omega)) \cap L^{\infty}\left([0, T) ; L^{\infty}(\Omega)\right) .
$$

Moreover, due to the previous bounds for $\theta_{\delta}$ and $\nabla \theta_{\delta}$, it is easy to verify that:

$$
\theta_{\delta}^{\prime} \in L^{2}\left([0, T) ; H^{-1}(\Omega)\right) .
$$

As a conclusion, we have:

$$
\theta_{\delta} \in L^{2}([0, T) ; H(\Omega)) \cap L^{\infty}\left([0, T) ; L^{\infty}(\Omega)\right) \text { and } \theta_{\delta}^{\prime} \in L^{2}\left([0, T) ; H^{-1}(\Omega)\right)
$$

\subsection{Existence of $\theta_{\delta}$}

We consider a Hilbert basis of the space $H(\Omega)$ and we apply the Faedo-Galerkin method. There exists a sequence $\left(\theta_{\delta}^{n}\right)_{n \in \mathbb{N}}$ solution of (34) in the vector space generated by the first $n$ vectors of the Hilbert basis. The a priori estimates are still valid for $\theta_{\delta}^{n}$. Therefore, the sequence $\left(\theta_{\delta}^{n}\right)_{n \in \mathbb{N}}$ is bounded in $L^{2}([0, T) ; H(\Omega)) \cap$ $L^{\infty}\left([0, T) ; L^{\infty}(\Omega)\right)$. Extracting sub-sequences, still written $\left(\theta_{\delta}^{n}\right)_{n \in \mathbb{N}}$, we find $\theta_{\delta} \in L^{2}([0, T) ; H(\Omega)) \cap L^{\infty}([0, T)$; $\left.L^{\infty}(\Omega)\right)$ such that:

- $\theta_{\delta}^{n} \longrightarrow \theta_{\delta}$ a.e. in $[0, T) \times \Omega$,

- $\theta_{\delta}^{n} \longrightarrow \theta_{\delta}$ weakly in $L^{2}\left([0, T) ; L^{2}(\Omega)\right)$,

- $\theta_{\delta}^{n} \longrightarrow \theta_{\delta}$ strongly in $L^{\infty}\left([0, T) ; L^{q}(\Omega)\right), \forall q<\infty$

- $\nabla \theta_{\delta}^{n} \longrightarrow \nabla \theta_{\delta}$ weakly in $L^{2}\left([0, T) ; L^{2}(\Omega)\right)$.

We will show that $\theta_{\delta}$ is a solution of the following equation:

$$
\frac{\partial \theta}{\partial t}+u \cdot \nabla \theta-\nabla \cdot\left(\left(\nu+c_{\theta} \frac{c_{\mu} l_{\mu} l_{\varepsilon}}{\sqrt{\theta^{2}+\delta^{2}}}\right) \nabla \theta\right)=-c_{\mu} \frac{l_{\mu}}{l_{\varepsilon}} P \theta|\theta|+\frac{1}{2} .
$$

Because of the convergences established previously and the results of the constant viscosity, the only term for which the convergence is difficult to prove, is $\nabla \cdot\left(\frac{l_{\mu} l_{\varepsilon}}{\sqrt{\theta^{2}+\delta^{2}}} \nabla \theta\right)$.

Actually, we have:

$$
I_{n}=\int_{0}^{T} \int_{\Omega}\left(\frac{l_{\mu} l_{\varepsilon}}{\sqrt{\left(\theta_{\delta}^{n}\right)^{2}+\delta^{2}}} \nabla \theta_{\delta}^{n}-\frac{l_{\mu} l_{\varepsilon}}{\sqrt{\left(\theta_{\delta}\right)^{2}+\delta^{2}}} \nabla \theta_{\delta}\right) \nabla \Phi,
$$

where $\Phi$ is a test function.

Let us remark that:

$$
\begin{aligned}
\frac{l_{\mu} l_{\varepsilon}}{\sqrt{\left(\theta_{\delta}^{n}\right)^{2}+\delta^{2}}} \nabla \theta_{\delta}^{n}-\frac{l_{\mu} l_{\varepsilon}}{\sqrt{\left(\theta_{\delta}\right)^{2}+\delta^{2}}} \nabla \theta_{\delta}= & \left(\frac{l_{\mu} l_{\varepsilon}}{\sqrt{\left(\theta_{\delta}^{n}\right)^{2}+\delta^{2}}}-\frac{l_{\mu} l_{\varepsilon}}{\sqrt{\left(\theta_{\delta}\right)^{2}+\delta^{2}}}\right) \nabla \theta_{\delta}^{n} \\
& -\frac{l_{\mu} l_{\varepsilon}}{\sqrt{\left(\theta_{\delta}\right)^{2}+\delta^{2}}}\left(\nabla \theta_{\delta}^{n}-\nabla \theta_{\delta}\right) .
\end{aligned}
$$


But, as $\delta^{2}+\theta_{\delta}^{2} \geq \delta^{2}$ and as $l_{\mu} l_{\varepsilon}$ is bounded, we have:

$$
\left|\int_{0}^{T} \int_{\Omega} \frac{l_{\mu} l_{\varepsilon}}{\sqrt{\left(\theta_{\delta}\right)^{2}+\delta^{2}}}\left(\nabla \theta_{\delta}^{n}-\nabla \theta_{\delta}\right) \nabla \Phi\right| \leq C \int_{0}^{T} \int_{\Omega}\left|\left(\nabla \theta_{\delta}^{n}-\nabla \theta_{\delta}\right) \nabla \Phi\right|,
$$

with $C$ depending on $\delta$, and we deduce:

$$
\lim _{n \rightarrow \infty} \int_{0}^{T} \int_{\Omega} \frac{l_{\mu} l_{\varepsilon}}{\sqrt{\left(\theta_{\delta}\right)^{2}+\delta^{2}}}\left(\nabla \theta_{\delta}^{n}-\nabla \theta_{\delta}\right) \nabla \Phi=0 .
$$

As $\Omega$ is a bounded set and as $l_{\mu} l_{\varepsilon}$ is bounded, we have, using the finite increment formula:

$$
\left|l_{\mu} l_{\varepsilon}\right|\left|\frac{1}{\sqrt{\left(\theta_{\delta}^{n}\right)^{2}+\delta^{2}}}-\frac{1}{\sqrt{\left(\theta_{\delta}\right)^{2}+\delta^{2}}}\right| \leq C\left|\theta_{\delta}^{n}-\theta_{\delta}\right|,
$$

with $C$ depending on $\delta$. Therefore:

$$
\begin{aligned}
\left|\int_{0}^{T} \int_{\Omega} l_{\mu} l_{\varepsilon}\left(\frac{1}{\sqrt{\left(\theta_{\delta}^{n}\right)^{2}+\delta^{2}}}-\frac{1}{\sqrt{\left(\theta_{\delta}\right)^{2}+\delta^{2}}}\right) \nabla \theta_{\delta} \nabla \Phi\right| & \leq C \int_{0}^{T} \int_{\Omega}\left|\theta_{\delta}^{n}-\theta_{\delta}\|\nabla \Phi\| \nabla \theta_{\delta}^{n}\right| \\
& \leq C\|\nabla \Phi\|_{L^{\infty}\left([0, T) ; L^{\infty}(\Omega)\right)} \int_{0}^{T} \int_{\Omega} \mid \theta_{\delta}^{n}-\theta_{\delta}\left\|\nabla \theta_{\delta}^{n}\right\| \\
& \leq C^{\prime} \int_{0}^{T}\left\|\theta_{\delta}^{n}-\theta_{\delta}\right\|_{L^{2}(\Omega)}\left\|\nabla \theta_{\delta}^{n}\right\|_{L^{2}(\Omega)} \\
& \leq C^{\prime}\left\|\nabla \theta_{\delta}^{n}\right\|_{L^{2}\left([0, T) ; L^{2}(\Omega)\right)}\left\|\theta_{\delta}^{n}-\theta_{\delta}\right\|_{L^{2}\left([0, T) ; L^{2}(\Omega)\right)}
\end{aligned}
$$

where $C^{\prime}=C\|\nabla \Phi\|_{L^{\infty}\left([0, T) ; L^{\infty}(\Omega)\right)}$. As $\nabla \theta_{\delta}^{n}$ is bounded in $L^{2}\left([0, T) ; L^{2}(\Omega)\right)$ by a constant independent of $n$ and as there is strong convergence of $\theta_{\delta}^{n}$ to $\theta_{\delta}$ in $L^{2}\left([0, T) ; L^{2}(\Omega)\right)$, we deduce:

$$
\lim _{n \rightarrow \infty} \int_{0}^{T} \int_{\Omega} l_{\mu} l_{\varepsilon}\left(\frac{1}{\sqrt{\left(\theta_{\delta}^{n}\right)^{2}+\delta^{2}}}-\frac{1}{\sqrt{\left(\theta_{\delta}\right)^{2}+\delta^{2}}}\right) \nabla \theta_{\delta} \nabla \Phi=0 .
$$

Consequently,

$$
\lim _{n \rightarrow \infty} I_{n}=0 .
$$

\subsection{Positivity of $\theta_{\delta}$}

Lemma 5.2. Under the hypotheses of Theorem 5.1, if $\theta_{0} \geq 0$, then $\theta_{\delta} \geq 0$ a.e. $x, t$.

Proof. The proof is the same as in the constant viscosity case, if one remarks that $l_{\mu} l_{\varepsilon} / \sqrt{\theta_{\delta}^{2}+\delta^{2}} \geq 0$ p.p. $x, t$.

\subsection{Another result on the regularity of $\theta_{\delta}$}

Remark 5.3. In order to prove the convergence of $\theta_{\delta}$ to $\theta$, solution of problem $(\mathcal{P})$, we have to demonstrate that $l_{\mu} l_{\varepsilon} / \theta$ is bounded, and in particular in the neighborhood of the wall. If we come back to the $k-\varepsilon$ variables, $l_{\mu} l_{\varepsilon} / \theta=l_{\mu} \sqrt{k}$ which is bounded near the wall since $k$ vanishes at the wall. Moreover, the physics of the flow says that the behavior of $l_{\varepsilon}$ is of order $y^{2} \sqrt{k}$ in the near-wall region. Therefore, $\theta$ behavior must be like that of a second degree polynomial. This is what we will check. 
From now on, we suppose that

$$
\left.\Omega=\prod_{i=1}^{3}\right] 0, a_{i}[
$$

where $a_{i}>0$ for $i=1,3$ and that $\left.\Gamma_{0}=\right] 0, a_{1}[\times\{0\} \times] 0, a_{3}[$ is the part of $\partial \Omega$ corresponding to the wall boundary. This is to simplify notations as $\forall M(X, Y, Z) \in \Omega, Y$ is the distance defined in (3) and as, in this case, the inward unit vector $\vec{n}$, normal to $\Gamma_{0}$ is constant. On $\Gamma_{1} \subset \partial \Omega$ such that $\Gamma_{0} \cup \Gamma_{1}=\partial \Omega$ (and $\Gamma_{0} \cap \Gamma_{1}=\emptyset$ ), we suppose that we have a homogeneous Neumann boundary condition for $\nabla \theta_{\delta}$. Of course, the previous results are still valid here.

Remark 5.4. Let us consider $y$ defined in relation (3). Actually, $y=\mathrm{d}\left(M, \Gamma_{0}\right)$ and $\Gamma_{0}$ is a flat surface; as a consequence, we have $\nabla\left(y^{2}\right)=2 y \vec{n}$ and $\triangle\left(y^{2}\right)=\nabla \cdot\left(\nabla\left(y^{2}\right)\right)=2 \nabla(y) \cdot \vec{n}=2>0$. This remark will be useful for the demonstration of the next lemma.

Lemma 5.5. Suppose that the hypotheses of Theorem 5.1 are true. Let $C$ be a positive constant such that:

$$
C c_{\mu} \frac{C_{1}}{C_{2}} M_{1} M_{2} b^{2}-\frac{1}{2}<0
$$

where $M_{1}=\|P\|_{L^{\infty}\left([0, T) ; L^{\infty}(\Omega)\right)}, M_{2}$ the uniform upper bound of $\theta_{\delta}$ in $L^{\infty}\left([0, T) ; L^{\infty}(\Omega)\right)$ and b an upper bound for the distance $y$ (defined in (3)). Then, if $\theta_{0}$ is such that $\theta_{0}-C y^{2} \geq 0$ a.e. on $\Omega$, we have:

$$
\theta_{\delta} \geq C y^{2} \text { p.p. } x, t
$$

where $\theta_{\delta}$ is solution of the following problem:

$$
\left\{\begin{array}{l}
\frac{\partial \Phi}{\partial t}-\nu \triangle \Phi=-c_{\mu} \frac{l_{\mu}}{l_{\varepsilon}} P|\Phi| \Phi+\frac{1}{2} \\
\Phi_{0}=\Phi(t=0)=\theta_{0}-C y^{2} \geq 0 \text { p.p. } x, t \\
\Phi=0 \text { on } \Gamma_{0} \\
\nabla \Phi \cdot \vec{n}=0 \text { on } \Gamma_{1} .
\end{array}\right.
$$

Proof. Let $C$ be a positive constant verifying (40) and $\psi=\theta_{\delta}-C y^{2}$. We will show that $\psi$, solution of the following problem, is positive:

$$
\left\{\begin{array}{l}
\frac{\partial \psi}{\partial t}-\nu \triangle \theta_{\delta}=-c_{\mu} \frac{l_{\mu}}{l_{\varepsilon}} P\left|\theta_{\delta}\right|\left(\psi+C y^{2}\right)+\frac{1}{2} \\
\psi_{0}=\psi(t=0)=\theta_{0}-C y^{2} \geq 0 \text { p.p. } x, t \\
\psi=0 \text { on } \Gamma_{0} .
\end{array}\right.
$$

Multiply equation in $\psi$ by $-\psi^{-}$and integrate in space. As $D=0, \nabla \theta_{\delta} \cdot \vec{n}=0$ on $\Gamma_{1}$ and $\psi^{-}=0$ on $\Gamma_{0}$, we have:

$$
\frac{1}{2} \frac{\mathrm{d}}{\mathrm{d} t} \int_{\Omega}\left(\psi^{-}\right)^{2}-\nu \int_{\Omega} \nabla \psi^{-} \nabla \theta_{\delta}=c_{\mu} \int_{\Omega} \frac{l_{\mu}}{l_{\varepsilon}} P\left|\theta_{\delta}\right|\left(\psi+C y^{2}\right) \psi^{-}-\frac{1}{2} \int_{\Omega} \psi^{-} .
$$

But, due to the definition of $C$, using obvious upper bounds for the positive variables and the previous remark, one easily sees that:

So

$$
c_{\mu} \int_{\Omega} \frac{l_{\mu}}{l_{\varepsilon}} P\left|\theta_{\delta}\right| C y^{2} \psi^{-}-\frac{1}{2} \int_{\Omega} \psi^{-} \leq 0 .
$$

$$
\frac{1}{2} \frac{\mathrm{d}}{\mathrm{d} t} \int_{\Omega}\left(\psi^{-}\right)^{2}-\nu \int_{\Omega} \nabla \psi^{-} \nabla \theta_{\delta} \leq 0
$$


As a consequence, using Gronwall's lemma and the fact that $\psi_{0}^{-}=0$,

$$
\psi^{-}=0 \text { a.e. } x, t
$$

if

$$
-\int_{\Omega} \nabla \psi^{-} \nabla \theta_{\delta} \geq 0
$$

Actually, the inequality (41) is true. We have:

$$
\begin{aligned}
-\int_{\Omega} \nabla \psi^{-} \nabla \theta_{\delta} & =-\int_{\Omega} \nabla \psi^{-} \nabla\left(\psi+C y^{2}\right) \\
& =-\int_{\Omega} \nabla\left(\psi^{+}-\psi^{-}+C y^{2}\right) \nabla \psi^{-} \\
& =\int_{\Omega}\left(\left(\nabla \psi^{-}\right)^{2}-C \nabla\left(y^{2}\right) \nabla \psi^{-}\right) \\
& \leq-C \int_{\Omega} \nabla\left(y^{2}\right) \nabla \psi^{-}
\end{aligned}
$$

As a consequence, we need the sign of the previous term. First, remark that:

$$
-\int_{\Omega} \nabla\left(y^{2}\right) \nabla \psi^{-}=\int_{\{\psi \leq 0\}} \nabla\left(y^{2}\right) \nabla \psi .
$$

But, thanks to Remark 5.4, one easily obtains, from the right hand side of relation (42), that:

$$
\begin{aligned}
\int_{\{\psi \leq 0\}} \nabla\left(y^{2}\right) \nabla \psi & =-\int_{\{\psi \leq 0\}} \triangle\left(y^{2}\right) \psi \\
& =-2 \int_{\{\psi \leq 0\}} \psi \\
& \geq 0 .
\end{aligned}
$$

Remark 5.6. We did not succeed in proving the same result in the case of the alternative problem $\left(\mathcal{P}_{\delta}\right)$, but we will suppose the existence of this constant $C$. As we will see, this result is essential for the convergence of $\theta_{\delta}$ to $\theta$, solution of $\mathcal{P}$.

\subsection{Convergence of $\theta_{\delta}$}

We have shown the convergence of $\theta_{\delta}^{n}$ to $\theta_{\delta}$, solution of $\left(\mathcal{P}_{\delta}\right)$. $\theta_{\delta}$ verifies the a priori estimates:

$$
\theta_{\delta} \in L^{2}([0, T) ; H(\Omega)) \cap L^{\infty}\left([0, T) ; L^{\infty}(\Omega)\right) \text { and } \theta_{\delta}^{\prime} \in L^{2}\left([0, T) ; H^{-1}(\Omega)\right)
$$

and there exists a constant $C$ strictly positive and independent of $\delta$ such that:

$$
\theta_{\delta} \geq C y^{2}
$$

Now, we have to pass to the limit when $\delta$ goes to 0 . Let $\left(\theta_{n}\right)_{n \in \mathbb{N}}$ be the sequence obtained when $\delta=1 / n$. There exists a sub-sequence that converges to a solution of $(\mathcal{P})$. 
As the a priori estimates guarantee that the bounds are independent of $\delta$, the sequence $\theta_{n}$ is bounded in $L^{2}([0, T) ; H(\Omega)) \cap L^{\infty}\left([0, T) ; L^{\infty}(\Omega)\right)$ and $\theta_{n}^{\prime}$ in $L^{2}\left([0, T) ; H^{-1}(\Omega)\right)$. We deduce from this point the existence of $\theta$ such that:

- $\theta_{n} \longrightarrow \theta$ a.e. in $[0, T) \times \Omega$, and therefore $\theta \geq 0$ a.e. $[0, T) \times \Omega$,

- $\theta_{n} \longrightarrow \theta$ weakly in $L^{2}\left([0, T) ; L^{2}(\Omega)\right)$,

- $\theta_{n} \longrightarrow \theta$ strongly in $L^{\infty}\left([0, T) ; L^{q}(\Omega)\right), \forall q<\infty$,

- $\nabla \theta_{n} \longrightarrow \nabla \theta$ weakly in $L^{2}\left([0, T) ; L^{2}(\Omega)\right)$.

From the weak convergence of $\theta_{n}$ to $\theta$ and of $\nabla \theta_{n}$ to $\nabla \theta$ in $L^{2}\left([0, T) ; L^{2}(\Omega)\right)$, one deduces:

$$
\begin{aligned}
& \int_{0}^{T} \int_{\Omega} \theta_{n}^{\prime} \Phi \longrightarrow \int_{0}^{T} \int_{\Omega} \theta^{\prime} \Phi, \forall \Phi \in \mathcal{D}([0, T) \times \Omega), \\
& \int_{0}^{T} \int_{\Omega} u \nabla \theta_{n} \Phi \longrightarrow \int_{0}^{T} \int_{\Omega} u \nabla \theta \Phi, \forall \Phi \in \mathcal{D}([0, T) \times \Omega), \\
& \nu \int_{0}^{T} \int_{\Omega} \nabla \theta_{n} \nabla \Phi \longrightarrow \nu \int_{0}^{T} \int_{\Omega} \nabla \theta \nabla \Phi, \forall \Phi \in \mathcal{D}([0, T) \times \Omega) .
\end{aligned}
$$

We have to demonstrate the convergence of the viscous and production terms.

For the production term, the demonstration is the same as for the sequence $\theta_{\delta}^{n}$ (Sect. 4.2 and Lem. 4.5). Finally, we have to prove:

$$
\int_{0}^{T} \int_{\Omega} \frac{l_{\mu} l_{\varepsilon}}{\sqrt{\theta_{n}^{2}+(1 / n)^{2}}} \nabla \theta_{n} \nabla \Phi-\int_{0}^{T} \int_{\Omega} \frac{l_{\mu} l_{\varepsilon}}{\theta} \nabla \theta \nabla \Phi \longrightarrow 0, \forall \Phi \in \mathcal{D}([0, T) \times \Omega)
$$

We decompose the substration in two terms:

$$
\frac{l_{\mu} l_{\varepsilon}}{\sqrt{\theta_{n}^{2}+(1 / n)^{2}}} \nabla \theta_{n}-\frac{l_{\mu} l_{\varepsilon}}{\theta} \nabla \theta=\frac{l_{\mu} l_{\varepsilon}}{\sqrt{\theta_{n}^{2}+(1 / n)^{2}}}\left(\nabla \theta_{n}-\nabla \theta\right)+\left(\frac{l_{\mu} l_{\varepsilon}}{\sqrt{\theta_{n}^{2}+(1 / n)^{2}}}-\frac{l_{\mu} l_{\varepsilon}}{\theta}\right) \nabla \theta .
$$

The convergence of the first term is assured. Actually, we have $\sqrt{\theta_{n}^{2}+(1 / n)^{2}} \geq \theta_{n} \geq C y^{2}$ where $C$ is defined in Lemma 5.5. This point leads to:

$$
\begin{aligned}
\left|\int_{0}^{T} \int_{\Omega} \frac{l_{\mu} l_{\varepsilon}}{\sqrt{\theta_{n}^{2}+(1 / n)^{2}}}\left(\nabla \theta_{n}-\nabla \theta\right) \nabla \Phi\right| & \leq \int_{0}^{T} \int_{\Omega}\left|\frac{l_{\mu} l_{\varepsilon}}{\sqrt{\theta_{n}^{2}+(1 / n)^{2}}}\right|\left|\left(\nabla \theta_{n}-\nabla \theta\right) \nabla \Phi\right| \\
& \leq \int_{0}^{T} \int_{\Omega} \frac{l_{\mu} l_{\varepsilon}}{C y^{2}}\left|\left(\nabla \theta_{n}-\nabla \theta\right) \nabla \Phi\right| \\
& \leq C^{\prime} \int_{0}^{T} \int_{\Omega}\left|\left(\nabla \theta_{n}-\nabla \theta\right) \nabla \Phi\right|,
\end{aligned}
$$

where $C^{\prime}$ is an upper bound of $l_{\mu} l_{\varepsilon} /\left(C y^{2}\right)$ that exists because of the definitions of $l_{\mu}, l_{\varepsilon}$ and because $\Omega$ is a bounded set. 
For the convergence of the last term, we use the regularity obtained in Lemma 5.5, which is still valid for $\theta$. Actually, we have:

$$
\begin{aligned}
\left|\frac{l_{\mu} l_{\varepsilon}}{\sqrt{\theta_{n}^{2}+(1 / n)^{2}}}-\frac{l_{\mu} l_{\varepsilon}}{\theta}\right| & \leq\left|\frac{l_{\mu} l_{\varepsilon}}{\theta_{n}}-\frac{l_{\mu} l_{\varepsilon}}{\theta}\right| \\
& \leq \frac{l_{\mu} l_{\varepsilon}}{\theta \theta_{n}}\left|\theta_{n}-\theta\right| \\
& \leq \frac{l_{\mu} l_{\varepsilon}}{C^{2} y^{4}}\left|\theta_{n}-\theta\right|,
\end{aligned}
$$

where $C$ is a constant obtained in Lemma 5.5.

Therefore, we have:

$$
\left|\int_{0}^{T} \int_{\Omega}\left(\frac{l_{\mu} l_{\varepsilon}}{\sqrt{\theta_{n}^{2}+(1 / n)^{2}}}-\frac{l_{\mu} l_{\varepsilon}}{\theta} \nabla \theta\right) \nabla \Phi\right| \leq \int_{0}^{T} \int_{\Omega} \frac{l_{\mu} l_{\varepsilon}}{C^{2} y^{4}}\left|\theta_{n}-\theta\right||\nabla \theta||\nabla \Phi|
$$

The strong convergence of $\theta_{n}$ to $\theta$ in $L^{2}\left([0, T) ; L^{2}(\Omega)\right)$ gives the result if $l_{\mu} l_{\varepsilon} / y^{4}$ is bounded. This term is bounded because of the definitions of $l_{\mu}$ and $l_{\varepsilon}$, as the function $f: y \longrightarrow l_{\mu} l_{\varepsilon} / y^{4}$ is continuous, defined on a closed and bounded interval and for $y=0$.

\section{Coupling the $\theta$ equation with the steady-State incompressible NAVIER-STOKES PROBLEM}

In this section, we are interested in the coupling between the steady-state Navier-Stokes system and our $\theta-$ or $\theta_{\delta}$ - turbulence model. We consider the following system:

$$
\left\{\begin{array}{l}
(u \cdot \nabla) u-\nabla \cdot\left(\left(\nu+c_{\theta} \nu_{t}\right) \nabla u\right)+\nabla p=f \text { on } \Omega \\
\nabla \cdot u=0 \text { on } \Omega \\
u \cdot \nabla \theta-\nabla \cdot\left(\left(\nu+c_{\theta} \nu_{t}\right) \nabla \theta\right)=-c_{\mu} \frac{l_{\mu}}{l_{\varepsilon}} P \theta+\frac{1}{2} \text { on } \Omega \\
u=0, \text { and } \theta=0 \text { on } \partial \Omega
\end{array}\right.
$$

where the turbulent cinematic viscosity $\nu_{t}$ is:

$$
\nu_{t}=c_{\mu} \frac{l_{\mu} l_{\varepsilon}}{\theta} \text { or } \nu_{t}=c_{\mu} \frac{l_{\mu} l_{\varepsilon}}{\sqrt{\theta^{2}+\delta^{2}}} .
$$

Our aim is to prove, if possible, the existence of a solution to this system. We will begin by proving the existence in a more general situation, as in [9]. In practice, we consider the following system:

$$
\left\{\begin{array}{l}
(u \cdot \nabla) u-\nabla \cdot(A(\theta) \nabla u)+\nabla p=f \text { on } \Omega \\
\nabla \cdot u=0 \text { on } \Omega \\
u \cdot \nabla \theta-\nabla \cdot(A(\theta) \nabla \theta)=-g(\theta, \nabla u) \theta+\frac{1}{2} \text { on } \Omega \\
u=0 \text { and } \theta=a \text { on } \partial \Omega
\end{array}\right.
$$

We make the following hypotheses on the data:

$\left(H_{1}\right) f \in H^{-1}(\Omega)^{N}$

$\left(H_{2}\right) a \geq 0$; 
$\left(H_{3}\right) A: \Omega \times \mathbb{R} \rightarrow \mathbb{R}^{N \times N}$ is a Caratheodory mapping. This means that $x \rightarrow A(x, s)$ is measurable in $\Omega, \forall s \in \mathbb{R}$ and $s \rightarrow A(x, s)$ is continuous in $\mathbb{R}$, a.e. in $\Omega$. Moreover, we suppose the existence of a scalar $\alpha>0$ such that

$$
A(x, s) \psi \psi \geq \alpha|\psi|^{2} \text {, for all } s \in \mathbb{R} \psi \in \mathbb{R}^{N} \text { and a.e. } x \in \Omega
$$

$\left(H_{4}\right)$ there exists a continuous function $\mathrm{d}: \mathbb{R} \rightarrow \mathbb{R}^{+}$such that

$$
A(x, s) \psi \psi \leq \mathrm{d}(s)|\psi|^{2} \text {, for all } s \in \mathbb{R} \psi \in \mathbb{R}^{N} \text { and a.e. } x \in \Omega \text {; }
$$

$\left(H_{5}\right) \mathrm{g}: \Omega \times \mathbb{R} \times \mathbb{R}^{N \times N} \rightarrow \mathbb{R}$ is a Caratheodory function and there exists $c \in L^{1}(\Omega)$ such that

$$
0 \leq \mathrm{g}(x, s, B) \leq \mathrm{d}(s)\left(c(x)+|B|^{2}\right), \text { for all } s \in \mathbb{R} B \in \mathbb{R}^{N \times N} \text { and a.e. } x \in \Omega .
$$

\subsection{Existence for a more general problem}

Theorem 6.1. Under hypotheses $\left(H_{1}-H_{5}\right)$, there exists $(u, \theta)$ solution of (44) such that:

$$
0 \leq \theta \leq a+\frac{C(N, \Omega)}{\alpha}, \text { a.e. in } \Omega,
$$

where $C(N, \Omega)$ is a constant value.

Proof. Let us begin by introducing the variational formulation of the generalized problem (44). We introduce the spaces $V=\left\{u \in H_{0}^{1}(\Omega)^{N}, \nabla \cdot u=0\right\}, W=H_{0}^{1}(\Omega) \cap L^{\infty}(\Omega)$ and $\langle.,$.$\rangle will represent the duality product$ between $V$ and $V^{\prime}$. The variational formulation of the problem is: find $u \in V, \theta \in H^{1}(\Omega)$ such that $\theta-a \in W$ and

$$
\left\{\begin{array}{l}
\int_{\Omega}(u \cdot \nabla) u v+\int_{\Omega} A(\theta) \nabla u \nabla v=\langle f, v\rangle, \forall v \in V \\
\int_{\Omega} u \nabla \theta \phi+\int_{\Omega} A(\theta) \nabla \theta \nabla \phi+\int_{\Omega} \theta g(\theta) \phi=\frac{1}{2} \int_{\Omega} \phi, \forall \phi \in W .
\end{array}\right.
$$

Of course, the pressure has disappeared, as all test functions are divergence-free. To recover $p$, we use the classical De Rham's argument [5]. Now, we apply the method presented in [9] which is based on Schauder's fixed point theorem. Let us introduce the set $B_{R}$ defined by:

$$
B_{R}=\left\{(\bar{u}, \bar{\theta}) \in L^{4}(\Omega)^{N} \times L^{2}(\Omega), 0 \leq \bar{\theta} \leq R,\|u\|_{L^{4}(\Omega)^{N}} \leq \frac{C_{0}(N, \Omega)}{\alpha}\|f\|_{H^{-1}(\Omega)^{N}}\right\},
$$

where $C_{0}(N, \Omega)$ is a new constant coming from the embedding of $H_{0}^{1}(\Omega)$ in $L^{4}(\Omega)$ (Sobolev embedding theorem). We define the operator $F:(\bar{u}, \bar{\theta}) \in B_{R} \rightarrow(u, \theta) \in L^{4}(\Omega)^{N} \times L^{2}(\Omega)$ by the following relations:

- Step 1: $u \in V=\left\{v \in H_{0}^{1}(\Omega)^{N}, \nabla \cdot v=0\right\}$ solution of:

$$
\int_{\Omega}(\bar{u} \cdot \nabla) u v+\int_{\Omega} A(\bar{\theta}) \nabla u \nabla v=\langle f, v\rangle, \forall v \in V .
$$

- Step 2: $\theta \in H^{1}(\Omega)$ such that $\theta-a \in W=H_{0}^{1}(\Omega) \cap L^{\infty}(\Omega)$ solution of:

$$
\int_{\Omega} \bar{u} \nabla \theta \phi+\int_{\Omega} A(\bar{\theta}) \nabla \theta \nabla \phi+\int_{\Omega} \theta g(\bar{\theta}, \nabla u) \psi=\frac{1}{2} \int_{\Omega} \psi, \forall \phi \in W .
$$


Step 1 is a transport Stokes system and has a unique solution by a simple application of the Lax-Milgram theorem. For Step 2, the solution exists thanks to the following lemma, established in [9]:

Lemma 6.2. Assume $A \in L^{\infty}(\Omega)^{N \times N}, u \in L^{2}(\Omega), \nabla \cdot u=0$ in $\Omega$ and $u \cdot n=0$ on $\partial \Omega, f \in L^{\infty}(\Omega)$ with $f \geq 0$ a.e. in $\Omega, h \in L^{1}(\Omega)$ with $h \geq 0$ a.e. in $\Omega$ and $a \in \mathbb{R}^{+}$. We suppose that there exists a constant $\alpha>0$ such that:

$$
A(x) \psi \psi \geq \alpha|\psi|^{2}, \forall \psi \in \mathbb{R}^{N} \text {, a.e. in } \Omega \text {. }
$$

Consider the following problem:

$$
\left\{\begin{array}{l}
z \in H^{1}(\Omega), z-a \in W \\
\int_{\Omega} A \nabla z \nabla \psi+\int_{\Omega} u \nabla z \psi+\int_{\Omega} h z \phi=\int_{\Omega} f \psi, \forall \psi \in W .
\end{array}\right.
$$

Then, there exists a unique solution $z$ to this problem and furthermore, we have:

$$
\left\{\begin{array}{l}
0 \leq z \leq a+\frac{C_{1}}{\alpha}\|f\|_{L^{\infty}(\Omega)} \text { a.e. in } \Omega \\
\|z\|_{H^{1}(\Omega)} \leq C_{2}, \forall \psi \in W
\end{array}\right.
$$

where $C_{1}=C_{1}(N, \Omega)$ and $C_{2}=C_{2}\left(a, \alpha, \Omega, N,\|f\|_{L^{\infty}(\Omega)},\|h\|_{L^{1}(\Omega)}\right)$.

Proof. One can refer to [9] for the proof of Lemma 6.2 .

From now on, we choose $R \geq a+C_{1} / 2 \alpha$. $F$ is well-defined. It is straightforward that $B_{R}$ is a non-empty, closed and convex set of $L^{4}(\Omega)^{N} \times L^{2}(\Omega)$. Moreover, $F$ is a continuous operator and $F\left(B_{R}\right) \subset B_{R}$. If we demonstrate that $F$ is compact, then, by Schauder's fixed point theorem, there will exist $(u, \theta)$ solution of problem (44). To prove the compactness of the operator, we use hypothesis $\left(H_{5}\right)$ to show that $g \in L^{1}(\Omega)$ and the bound given by Lemma $6.2[9]$.

\subsection{Applications to our cases}

It is obvious that the method can be applied without modification to each steady-state version of the $\theta_{\delta}$ equation (from problem $\left(\mathcal{P}_{\delta}\right)$ ) coupled with the steady-state Navier-Stokes equations, as all the hypotheses $\left(H_{1}-H_{5}\right)$ are true (we take $\left.A=\left(\nu+c_{\theta} c_{\mu} l_{\mu} l_{\varepsilon} / \sqrt{\theta^{2}+\delta^{2}}\right) I\right)$.

Remark 6.3. If we neglect the transport terms in (45) in order to obtain the Stokes problem coupled with our $\theta_{\delta}$ equation, we can demonstrate, as in [9], the existence of the solution if the hypotheses $\left(H_{1}-H_{5}\right)$ are true. The demonstration is also based on Schauder's fixed point theorem and on compactness.

Actually, the application of the method to the original problem (43) raises some difficulties.

First, if we want to apply the previous method (Schauder's fixed point theorem) to the original equation (33), $P=|\nabla u|^{2}$ must be bounded uniformly (in $L^{\infty}(\Omega)$ ) with respect to $\delta$, and the inequality $\theta_{\delta} \geq C y^{2}$ is a necessary conclusion of Lemma 6.2. We did not succeed in proving this inequality for the evolution problem with matrix $A$, and we did not succeed anymore in proving it for the time-independent problem; moreover, we are unable to prove the result for $P$. Another method would consist in solving the problem $\left(\mathcal{P}_{\delta}\right)$ coupled with the steady-state Navier-Stokes equations, and passing to the limit. Here also, the difficulty comes from the same defects of $P$ and $\theta_{\delta}$ (existence of constant $C$ ).

\section{Coupling the $\theta$ equation with the incompressible Navier-Stokes problem}

In this section, we wish to investigate the coupling between our $\theta_{\delta}$ turbulence model and the Navier-Stokes equations for incompressible flows. For 2D and 3D cases, we will prove the existence of a solution for the coupled problem, neglecting the transport terms, while, in $2 \mathrm{D}$, we will prove the existence of a solution for the complete 
problem. As we will see, obtaining existence of a solution to these problems will be straightforward. We will only need some theorems from $[8]$.

\subsection{Incompressible Navier-Stokes and $\theta$ equations without transport terms}

In the following, we consider a bounded domain $\Omega$ with Lipschitz boundary and define the spaces:

$Q=\Omega \times[0, T)$,

$H=\left\{v \in L^{2}(\Omega)^{N}, \nabla \cdot v=0\right.$, and $\left.v \cdot n=0\right\}, V=\left\{v \in H_{0}^{1}(\Omega)^{N}, \nabla \cdot v=0\right\}, V^{\prime}=$ dual space of $V$,

$W_{1}=\left\{v \in L^{2}([0, T) ; V), v^{\prime} \in L^{2}\left([0, T) ; V^{\prime}\right)\right\}$,

$W_{2}^{q}=\left\{v \in L^{2}\left([0, T) ; H_{0}^{1}(\Omega)\right), v^{\prime} \in L^{2}\left([0, T) ; W^{-1, q}(\Omega)\right), 1<q \leq \infty\right.$ and

$W_{3}^{q}=\left\{v \in L^{q}\left([0, T) ; W_{0}^{1, q}(\Omega)\right), v^{\prime} \in L^{1}\left([0, T) ; W^{-1, q}(\Omega)\right)\right\}, 1<q \leq \infty$.

We want to solve the following system, which comes from the weak formulation of the classical Navier-Stokes equations:

$$
\left\{\begin{array}{l}
\int_{0}^{T}\left\langle u^{\prime}, v\right\rangle+\int_{Q} A(\theta) \nabla u \nabla v=\int_{0}^{T}\langle f, v\rangle, \forall v \in L^{2}([0, T) ; V) \\
\int_{0}^{T}\left\langle\theta^{\prime}, \phi\right\rangle+\int_{Q} A(\theta) \nabla \theta \nabla \phi=\int_{Q}\left(\frac{1}{2}-c_{\mu} \frac{l_{\mu}}{l_{\varepsilon}}|\nabla u|^{2}\right) \phi, \forall \phi \in \mathcal{D}(Q) \\
u(x, 0)=u_{0}, \theta(x, 0)=\theta_{0} \text { in } \Omega \\
\theta(x, t)=a \text { on } \partial \Omega \times(0, T) .
\end{array}\right.
$$

As previously, $\langle.,$.$\rangle represents the duality product between V$ and $V^{\prime}$. The existence of a solution to this problem, with respect to the behavior of the initial condition, is given by the following theorem, proved in [8]:

Theorem 7.1. We assume the following hypotheses:

$\left(H_{1}\right) f \in L^{2}\left([0, T) ; H^{-1}(\Omega)^{N}\right), u_{0} \in H$;

$\left(H_{2}\right) a \geq 0$

$\left(H_{3}\right) \theta_{0} \geq 0$

$\left(H_{4}\right) A: Q \times \mathbb{R} \rightarrow \mathbb{R}^{N \times N}$ is a Caratheodory matrix function and there exists a constant $\alpha>0$ and a non decreasing function $d: \mathbb{R}^{+} \rightarrow \mathbb{R}^{+}$such that:

$$
\alpha|\psi|^{2} \leq A(x, t, s) \psi \psi \leq d(|s|)|\psi|^{2} \text {, a.e. in } Q, \forall s \in \mathbb{R} .
$$

Under hypotheses $\left(H_{1}-H_{4}\right)$, if $\theta_{0} \in L^{\infty}(\Omega)$, then there exists $(u, \theta)$ solution of (46) such that

$$
\begin{gathered}
u \in W_{1}, \theta-a \in W_{2}^{q} \cap L^{\infty}(\Omega), \forall q \in\left(1, \frac{N}{N-1}\right), \\
0 \leq \theta(x, t) \leq \max \left\{\left\|\theta_{0}\right\|_{L^{\infty}(\Omega)}, a\right\}+t, \text { a.e. in } Q .
\end{gathered}
$$

If we assume hypotheses $\left(H_{1}-H_{4}\right)$ and also that d defined in $\left(H_{4}\right)$ is constant, if $\theta_{0} \in L^{1}(\Omega)$, then there exists $(u, \theta)$ solution of $(46)$ such that:

$$
\begin{gathered}
u \in W_{1}, \theta-a \in W_{3}^{q} \cap L^{\infty}\left([0, T) ; L^{1}(\Omega)\right), \forall q \in\left(1, \frac{N+2}{N+1}\right), \\
\theta \geq 0 \text { a.e. in } Q \text { and } \theta^{2}|\nabla u|^{2} \in L^{1}(Q) .
\end{gathered}
$$


Proof. One can refer to [8] for the demonstration, which can easily be extended to (46).

As a consequence, due to our matrix $A$, we have existence of the solution for both $L^{\infty}$ and $L^{1}$ initial conditions when we consider the $\theta_{\delta}$ equation (34) coupled with the incompressible Navier-Stokes equations:

$$
\nu|\psi|^{2} \leq\left(\nu+c_{\theta} \frac{c_{\mu} l_{\mu} l_{\varepsilon}}{\sqrt{\theta_{\delta}^{2}+\delta^{2}}}\right) I \psi \psi \leq\left(\nu+c_{\theta} \frac{c_{\mu} B}{\delta}\right)|\psi|^{2},
$$

where $B$ is an upper bound of $l_{\mu} l_{\varepsilon}$. To pass to the limit when $\delta$ goes to 0 , we need $P=|\nabla u|^{2}$ to be in $L^{\infty}\left([0, T) ; L^{\infty}(\Omega)\right)$. As previously, we did not succeed in proving such a result and a consequence of this point is the lack of information to prove the existence of the constant $C$ (Lem. 5.5).

\subsection{Incompressible Navier-Stokes equations coupled with our $\theta$ turbulence model}

In this section, we consider the complete Navier-Stokes equations, i.e. we do not neglect the transport terms. In this case, a new theorem about the coupling of these equations has been proved in [8]:

Theorem 7.2. Let the dimension space $N$ be 2 and assume hypotheses $\left(H_{1}-H_{4}\right)$. If $\theta_{0} \in L^{\infty}(\Omega)$ then there exists $(u, \theta)$ solution of the problem (47) and we have:

$$
\begin{gathered}
u \in W_{1}, \theta-a \in W_{2}^{q} \cap L^{\infty}(\Omega), \forall q \in\left(1, \frac{N}{N-1}\right), \\
0 \leq \theta(x, t) \leq \max \left\{\left\|\theta_{0}\right\|_{L^{\infty}(\Omega)}, a\right\}+t, \text { a.e. in } Q .
\end{gathered}
$$

If $\theta_{0} \in L^{1}(\Omega)$ and we assume hypothesis $\left(H_{5}\right)$ then there exists $(u, \theta)$ solution of the problem $(47)$ and we have:

$$
\begin{gathered}
u \in W_{1}, \theta-a \in W_{3}^{q} \cap L^{\infty}\left([0, T) ; L^{1}(\Omega)\right), \forall q \in\left(1, \frac{N+2}{N+1}\right), \\
\theta \geq 0 \text { a.e. in } Q \text { and } \theta^{2}|\nabla u|^{2} \in L^{1}(Q) .
\end{gathered}
$$

Problem (47) is the following:

$$
\left\{\begin{array}{l}
\int_{0}^{T}\left\langle u^{\prime}, v\right\rangle+\int_{Q}(u \cdot \nabla) u v+\int_{Q} A(\theta) \nabla u \nabla v=\int_{0}^{T}\langle f, v\rangle, \forall v \in L^{2}([0, T) ; V), \\
\int_{0}^{T}\left\langle\theta^{\prime}, \phi\right\rangle-\int_{Q} u \nabla \psi \theta+\int_{Q} A(\theta) \nabla \theta \nabla \phi=\int_{Q}\left(\frac{1}{2}-c_{\mu} \frac{l_{\mu}}{l_{\varepsilon}}|\nabla u|^{2}\right) \phi, \forall \phi \in \mathcal{D}(\mathcal{Q}) \\
u(x, 0)=u_{0}, \theta(x, 0)=\theta_{0} \text { in } \Omega \\
\theta(x, t)=a \text { on } \partial \Omega \times(0, T)
\end{array}\right.
$$

and comes from the weak formulation of this system:

$$
\left\{\begin{array}{l}
u^{\prime}+(u \cdot \nabla) u-\nabla \cdot(A(\theta) \nabla u)+\nabla p=f, \nabla \cdot u=0 \text { in } Q \\
\theta^{\prime}+u \nabla \theta-\nabla \cdot(A(\theta) \nabla \theta)=\frac{1}{2}-c_{\mu} \frac{l_{\mu}}{l_{\varepsilon}}|\nabla u|^{2}|\theta| \theta \text { in } Q \\
u(x, 0)=u_{0}, \theta(x, 0)=\theta_{0} \text { in } \Omega \\
\theta(x, t)=a \text { on } \partial \Omega \times(0, T) .
\end{array}\right.
$$

Proof. One can refer to [8] for a demonstration which can easily be extended to (47).

Of course, this shows the existence of the solution for the Navier-Stokes equations coupled with the $\theta_{\delta}$ equation (34). As previously, we did not succeed in proving such a result for the original $\theta$ turbulence model. 


\section{Numerical applications of the New $\theta-$ And $\theta_{\delta}-$ MOdels}

Now, we are interested in the numerical applications of our new turbulence model. First, the question is to know how to determine the constant $c_{\theta}$.

\subsection{Determination of $c_{\theta}$}

The principle for the determination of the constants of the $k-\varepsilon$ model can be found in [16]. The arguments are physical ones and are based on the study of the variables near solid walls with a reduction of scales.

We suppose that $\Omega$ is an open set of $\mathbb{R}^{3}$ of the form presented in (39) with a solid boundary on the flat surface $\left.\Gamma_{0}=\right] 0, a_{1}[\times\{0\} \times] 0, a_{3}[$. As previously, this domain is interesting as the distance between a point $M(X, Y, Z)$ in the flow and the wall boundary $\Gamma_{0}$ is $Y$. Moreover, physicists have shown that in the boundary layer over a flat plate, we can neglect the variations of the variables in the tangential directions. This means that $\partial / \partial x \ll \partial / \partial y$ and $\partial / \partial z \ll \partial / \partial y$. We assume that the velocity field is parallel to the wall boundary and that $\theta$ is stationary. As a consequence, we have:

$$
\begin{gathered}
u \cdot \nabla \theta=0 \\
\nabla \cdot\left(\left(\nu+c_{\theta} \frac{c_{\mu} l_{\mu} l_{\varepsilon}}{\theta}\right) \nabla \theta\right)=\frac{\partial}{\partial y}\left(\left(\nu+c_{\theta} \frac{c_{\mu} l_{\mu} l_{\varepsilon}}{\theta}\right) \frac{\partial \theta}{\partial y}\right) \\
P=\left(\frac{\partial u}{\partial y}\right)^{2} .
\end{gathered}
$$

Near solid walls, there may be a boundary layer, for which experimental observations have shown logarithmic profile for the flow velocity [3]:

$$
\frac{u}{u_{\tau}}=\frac{1}{\kappa} \ln \left(y^{+}\right)+5.5
$$

where $\kappa, y^{+}$and $u_{\tau}$ are defined in Section 1 and $u$ is the tangential component of the velocity. Moreover, experimental studies have also shown that relation (48) is valid when $50 \leq y^{+} \leq 200$ [3]. As a consequence, the $\theta$ model equation becomes:

$$
-\frac{\partial}{\partial y}\left(c_{\theta} \frac{c_{\mu} l_{\mu} l_{\varepsilon}}{\theta} \frac{\partial \theta}{\partial y}\right)=-c_{\mu} \frac{l_{\mu}}{l_{\varepsilon}} P \theta^{2}+\frac{1}{2} .
$$

Physical studies enable us to admit that the $k$ equation (7) is satisfied by the following variable:

$$
k=\frac{u_{\tau}^{2}}{\sqrt{c_{\mu}}}
$$

We deduce that the $\theta$ equation is satisfied by:

$$
\theta=\frac{l_{\varepsilon}}{\sqrt{k}}=\frac{l_{\varepsilon} \sqrt[4]{c_{\mu}}}{u_{\tau}} \text { and } P=\frac{u_{\tau}^{2}}{\kappa^{2} y^{2}} .
$$

So, with the hypotheses, when $50 \leq y^{+} \leq 200$, the $\theta$ equation becomes:

$$
-c_{\theta} c_{\mu} \frac{u_{\tau}}{\sqrt[4]{c_{\mu}}}\left(\frac{\partial l_{\mu}}{\partial y} \frac{\partial l_{\varepsilon}}{\partial y}+l_{\mu} \frac{\partial^{2} l_{\varepsilon}}{\partial y^{2}}\right)=-c_{\mu}^{5 / 4} \frac{l_{\mu} l_{\varepsilon}}{\kappa^{2} y^{2}}+\frac{1}{2} .
$$

This relation expresses that $c_{\theta}$ is not constant and is a positive function of $y^{+}$. We extend $c_{\theta}$ with the value of $c_{\theta}(50)$ when $y^{+}<50$. In Figure 1, we have plotted the behavior of $\theta$ extracted from its definition (50). 


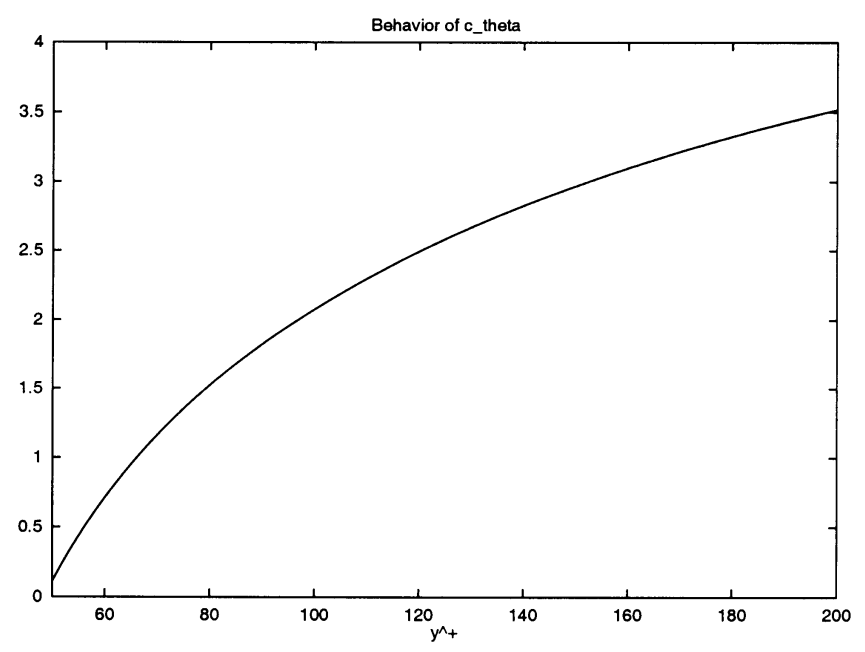

Figure 1. Behavior of $c_{\theta}$; for $y^{+}<50$, we choose $c_{\theta}\left(y^{+}\right)=c_{\theta}(50)$.

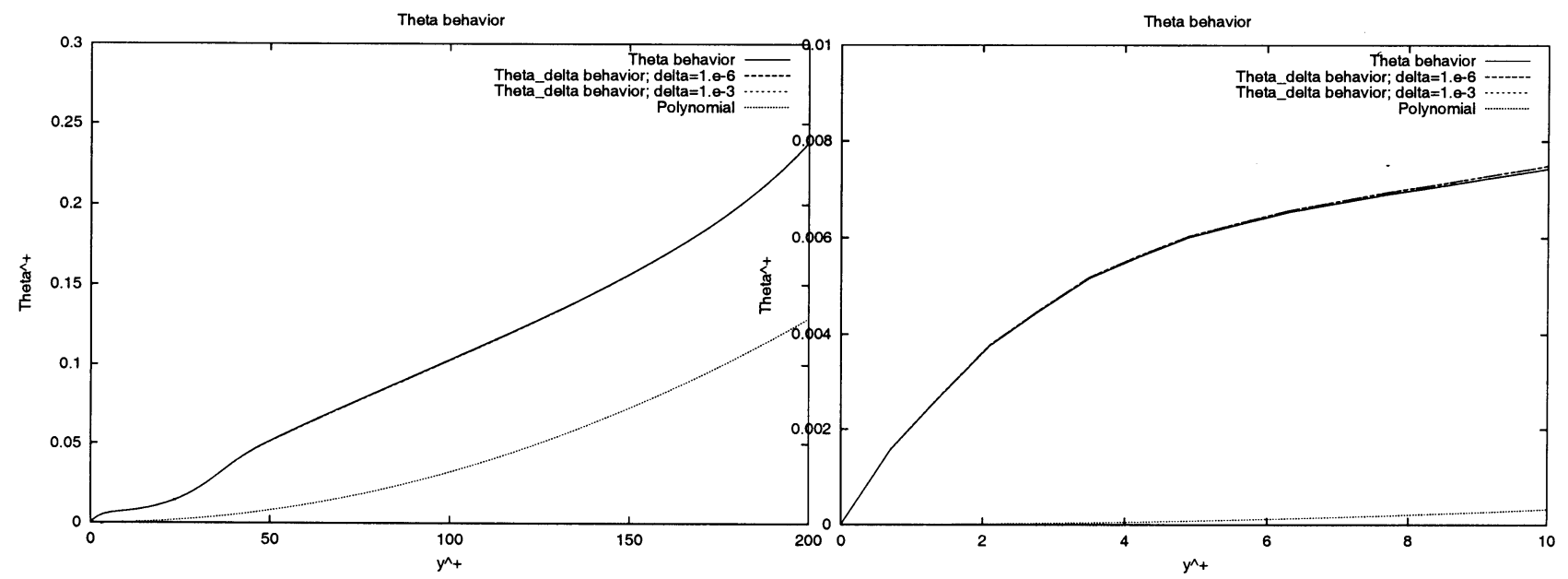

Figure 2. Behavior of $\theta$ and $\theta_{\delta}$ in the boundary-layer (left) and zoom on the near-wall region (right). On both figures, we also show the polynomial $C\left(y^{+} \nu / u_{\tau}\right)^{2}=C y^{2}$ (Lem. 5.5) and the behavior of $\theta_{\delta}$ for $\delta=10^{-3}$ and $\delta=10^{-6}$. The alternative model gives the same solution as the original model in regions away from the wall and behaves well closed to the wall (where the original model would fail without an a priori information on $\nu_{t}$ ).

\subsection{Assumption for the constant $C$ (Lem. 5.5)}

In Figure 2, we present the results obtained from the computation of $\theta$ in the near wall region, without neglecting the turbulent viscosity contribution. The Reynolds number is $10^{7}$, which corresponds to the laminar viscosity $\nu=10^{-7}$ and the non dimension friction velocity $u_{\tau} / u_{\infty}$ is 0.01 . The problem is to evaluate the production term $P$. For a flat plate, physicists [3] have shown that the velocity profile is linear when $y^{+} \leq 5$ : $u=u_{\tau} y^{+}$and logarithmic (48) for $50 \leq y^{+} \leq 200$. We can evaluate $\partial u / \partial y$ in those regions and we use a linear interpolation in the buffer one $\left(5<y^{+}<50\right)$. The constant $C$ found in Lemma 5.5 is used for the plotting of the polynomial: this is the proof that our assumption is not false, even if we did not succeed in proving the 
result for the complete problem $\left(\mathcal{P}_{\delta}\right)$. Moreover, the good fit between the $\theta-$ and $\theta_{\delta}-$ behaviors is another argument for choosing the $\theta_{\delta}$ turbulence model instead of the original one.

\section{IMPLEMENTATION OF THESE MODELS IN A SOLVER}

In this section, we will describe the technique to use for the implementation of the previous models. In particular, we will explain why the $\theta_{\delta}$ model is numerically more interesting that the $\theta$ model.

For the implementation, the difficulty is to account for the boundary condition, and especially, as seen before, for the wall turbulent viscosity. For the $\theta$ model, we have to impose in a strong form that $\nu_{t}=0$ on the boundary. This hypothesis comes from the polynomial property of $\theta$. However, it is not necessary for the $\theta_{\delta}$ model, which implies an easier implementation in existing codes. Moreover, because of our mathematical work, the $\theta_{\delta}$ model may be preferred to the original one. More sophisticated numerical simulations with an extension of this model for compressible flows have been performed for various high-speed configurations [14].

The principle of the implementation is to make a splitting between diffusion and transport parts of the $\theta$ or $\theta_{\delta}$ equations. Indeed, let $\theta_{n}$ be the value of $\theta$ or $\theta_{\delta}$ at step $n$ (previous iteration). If we write $\Delta t$ for the time step, we compute $\theta_{n+1 / 2}$ as follows:

$$
\left(\frac{1}{\Delta t}+u^{n} \nabla .+\frac{\left(S_{\theta}^{-}\right)_{n}}{\theta_{n}}\right) \theta_{n+1 / 2}=\frac{\theta_{n}}{\Delta t}+\left(S_{\theta}^{+}\right)_{n}
$$

where $\left(S_{\theta}^{-}\right)_{n}$ and $\left(S_{\theta}^{+}\right)_{n}$ are respectively the negative and positive parts of the right-hand side of the $\theta-$ or $\theta_{\delta}-$ equation. Then, we take into account the viscous part. This algorithm is unconditionally stable and guarantees the positivity of $\theta$ or $\theta_{\delta}$ if the vertices of the mesh do not present any blunt angle.

\section{Conclusion}

A mathematical study has been performed for existence and positivity of the two-layer $k-\varepsilon$ model. The necessary conditions for existence and positivity of the solution of the model have been identified. This permits to establish a required framework for the incoming models and this also helps for choosing the one with the best mathematical and numerical behavior between existing turbulence models. In addition, as our aim is to develop wall functions, we needed to identify the most mathematically suitable two-layer model to be used as starting point for this development. Finally, an alternative $\theta_{\delta}$ model may be chosen as it shows more regularity and less numerical implementation difficulties and leads to existence of a solution for the Navier-Stokes problem coupled with the turbulence model equation.

Acknowledgements. The authors gratefully acknowledge the many helpful suggestions of O. Anza Hafsa, V. Rougeot, J. Bolte and P. Redont during the preparation of the paper.

\section{REFERENCES}

[1] S. Clain, Analyse mathématique et numérique d'un modèle de chauffage par induction. Ph.D. Thesis, École Polytechnique Fédérale de Lausanne (1994).

[2] S. Clain and R. Touzami, Solution of a two-dimensional stationary induction heating problem without boundedness of the coefficients. RAIRO Modél. Math. Anal. Numér. 31 (1997) 845-870.

[3] J. Cousteix, Turbulence et couche limite. Cepadues, Ed., Toulouse (1990).

[4] R. Dautrey and J.-L. Lions, Analyse mathématique et calcul numérique pour les sciences et les techniques. Vol. 8. Masson, Ed., Paris (1988).

[5] G. de Rham, Variétés différientiables. Hermann, Paris (1960).

[6] T. Gallouët and R. Herbin, Existence of a solution to a coupled elliptic system. Appl. Math. Lett. 2 (1994) 49-55.

[7] T. Gallouët, J. Lederer, R. Lewandowski, F. Murat and L. Tartar, On a turbulent system with unbounded eddy viscosities. To appear in J. Non-Linear Anal. TMA. 
[8] M. Gómez Mármol and F. Ortegón Gallego, Existence of Solution to Non-Linear Elliptic Systems Arising in Turbulence Modelling. $M^{3} A S$ (Math. Models Methods Appl. Sci.) 10 (2000) 247-260.

[9] M. Gómez Mármol and F. Ortegón Gallego, Coupling the Stokes and Navier-Stokes Equations with Two Scalar Nonlinear Parabolic Equations. ESAIM: M2AN 33 (1999) 157-167

[10] R. Lewandowski and B. Mohammadi, Existence and Positivity Results for the $\phi-\theta$ and a Modified $k-\varepsilon$ Turbulence Models. $M^{3} A S$ (Math. Models Methods Appl. Sci.) 3 (1993) 195-215.

[11] R. Lewandowski, Analyse mathématique et océanographie. Masson, Ed., Paris (1997).

[12] R. Lewandowski, The mathematical analysis of the coupling of a turbulent kinetic energy equation to the Navier-Stokes equation with an eddy viscosity. J. Non-Linear Anal. TMA 28 (1997) 393-417.

[13] J.-L. Lions, Quelques méthodes de résolution des problèmes aux limites non linéaires. Gauthier-Villard, Eds., Dunod, Paris (1969).

[14] B. Mohammadi and G. Puigt, Generalized Wall Functions for High-Speed Separated Flows over Adiabatic and Isothermal Walls. To appear in Internat. J. Comput. Fluid Dyn.

[15] B. Mohammadi, A Stable Algorithm for the $k-\varepsilon$ Model for Compressible Flows. INRIA, Report No. 1335 (1990).

[16] B. Mohammadi and O. Pironneau, Analysis of the $k-\varepsilon$ turbulence model. Wiley-Masson, Eds., Paris (1994).

[17] V.C. Patel, W. Rhodi and G. Scheuerer, Turbulence models for near-wall and low-Reynolds number flows: a review. AIAA J. 23 (1984) 1308-1319.

[18] R. Temam, Infinite Dimensional Systems in Mechanics and Physics. 2nd edn., Springer-Verlag, Eds., Berlin, Heidelberg, New York (1997).

To access this journal online:

www.edpsciences.org 